\title{
Backscatter Wireless Communications and Sensing in Green Internet of Things
}

\author{
Usman Saleh Toro, Student Member, IEEE, Kaishun Wu, Senior Member, IEEE, and Victor C. M. Leung, Life \\ Fellow, IEEE
}

\begin{abstract}
Internet of Things (IoT) is a key technology for enabling ubiquitous applications that interconnect with cyberphysical systems in various environments. However, its large scale adoption is strongly impeded by the limited energy available for most IoT devices that are battery-powered, and further challenged by the growing demands to pack increasing functionalities into IoT devices while shrinking their sizes. To address these problems, researchers have developed techniques for energy harvesting, wireless power transfer, and minimizing power consumption in the sensing, communication and computation components of IoT nodes, as found in many surveys. In contrast, this paper surveys Backscatter Communication (BackCom), a recently emerged technique that enables green IoT through joint wireless communication and sensing and potentially allows IoT devices to operate without batteries. The operating principle of BackCom-based green IoT, its architecture and evolution are presented. Also state-of-the-art applications such as healthcare, agriculture, human activity recognition, transportation and mobile IoT are reviewed together with the operational and security challenges faced by these applications and potential solution techniques to address these challenges while ensuring a high energy efficiency. Lastly, some future applications of BackCombased green IoT are discussed.
\end{abstract}

Index Terms-Green Internet of Things, Wireless Communications, Sensing, Backscatter Communications

\section{INTRODUCTION}

$\mathbf{T}$ HE adoption of Internet of Things (IoT) devices has redefined the process of sensing, storage and exchange of information in simple environments such as homes and offices [1]. More importantly, IoT enables complex environments such as power grids [2], transportation networks [3] and disaster scenes [4] to deploy a large number of networked sensors that collect large amounts of data and send them to computing servers for analysis. Such networked cyberphysical systems lead to better decision making, more efficient system operations and improved safety of the environments. Transforma Insights predict that adoption of IoT devices will increase to 24.1 billion and revenue generated from IoT will reach 1.5 trillion USD by 2030 [5]. This forecast will largely depends on success in minimizing energy demands of those devices. Therefore, rapid development and implementation of green techniques are required to achieve these forecasts.

For better comprehension, abbreviations used in the paper and their meanings are listed in Table I.

The authors are with the College of Computer Science and Software Engineering, Shenzhen University, Shenzhen 518060, Kaishun Wu is the corresponding author (email: \{torousmansaleh2019@email.szu.edu.cn, wu@szu.edu.cn,vleung@iee.org $\}$ ).

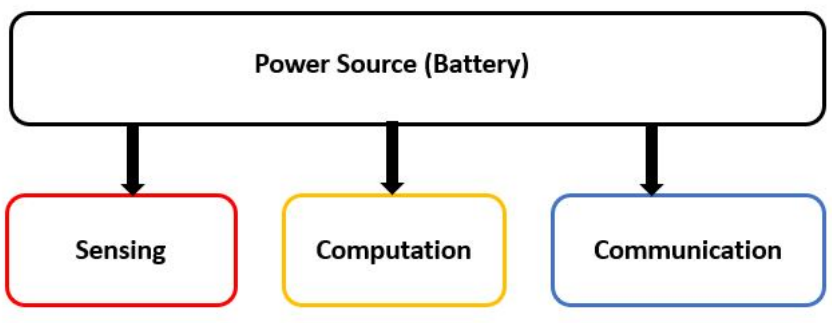

Figure 1: Components of an IoT Node

Recently, IoT devices have been increasingly leveraged for various applications while their sizes are becoming smaller. Miniaturization leads to smaller batteries and hence less available energy for longer life times. In order to efficiently manage the low power available, energy efficient (green) techniques have been developed to reduce power consumption at the different components of an IoT sensor node [6], [7], [8], including sensing, computation and communication components as shown in Figure 1. In addition to greening the components of an IoT node, energy harvesting (EH) [9], low power cryptography [10] and wireless power transfer (WPT) techniques [11] have been developed for extending the operating time of battery-powered IoT devices. Despite success shown by these green techniques, they do not provide a single infrastructure that jointly senses data and communicates the sensed data to a receiver for processing at very low power budgets. Fortunately, advances in edge computing have allowed computation of IoT data to be relegated to data centres and smart phones [12] leaving communication and sensing to be the major energy consuming components of an IoT node. Designed to significantly improve the power efficiency of sensing and communication jointly, Backscatter Communication (BackCom) is

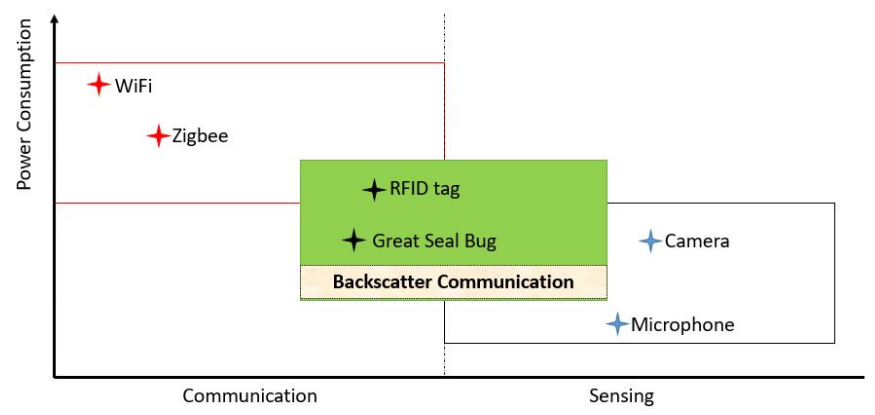

Figure 2: Joint Communication and Sensing with Backscatter Communication 
Table I: Abbreviations used and their meanings

\begin{tabular}{|c|c|c|c|}
\hline Abbreviation & Meaning & Abbreviation & Meaning \\
\hline $\mathbf{A P}$ & Access Point & AmBC & Ambient Backscatter Communication \\
\hline ASK & Amplitude Shift Keying & ADC & Analog-to-digital Converter \\
\hline BackCom & Backscatter Communication & BC-NOMA & Backscatter Cooperation-NOMA \\
\hline BRN & Backscatter relaying Network & BS & Base Station \\
\hline BPSK & Binary Phase Shift Keying & BER & Bit-error-rate \\
\hline CSI & Channel State Information & CMA & Characteristic Mode Analysis \\
\hline COTS & Commercial off-the-shelf & DL & Deep Learning \\
\hline DRL & Deep RL & D2D & device-to-device \\
\hline DBC & Digital Baseband Controller & DAC & Digital-to-analog Converter \\
\hline DC & $\begin{array}{l}\text { Direct Current } \\
\text { Diret }\end{array}$ & EE & $\begin{array}{l}\text { Energy Efficient } \\
\text { E }\end{array}$ \\
\hline EH & Energy Harvesting & ET & Energy Transmitter \\
\hline ER & Energy Receiver & $\mathbf{E M}$ & Expectation Maximization \\
\hline FM & Frequency Modulation & FAP & Full duplex AP \\
\hline IRS & Intelligent Reflective Surface & IoT & Internet of Things \\
\hline IoWT & Internet of Wearable Things & LoRA & Long range Radio \\
\hline MTC & Machine-type communication & MAC & Medium Access Control \\
\hline MAV & Micro Aerial Vehicle & MIMO & multiple-input multiple-output \\
\hline M-MAC & Multiplicative-multiple Access Channel & NIC & Network Interface Card \\
\hline NOMA & Non-orthogonal Multiple Access & OOK & on-off Keying \\
\hline OFDMA & Orthogonal Frequency Division Multiple Access & OFDM & Orthogonal Frequency Division Multiplexing \\
\hline OMA & Orthogonal Multiple Access & PAM & \\
\hline PD-NOMA & Power-Domain NOMA & QAM & Quadrature Amplitude Modulation \\
\hline QoS & Quality of Service & RFID & Radio Frequency Identification \\
\hline $\mathbf{R F}$ & Radio Frequency & RL & Reinforcement Learning \\
\hline SIC & Successive Interference Cancellation & TDMA & Time Division Multiple Access \\
\hline UAV & Unmanned Aerial Vehicle & V2I & vehicle-to-infrastructure \\
\hline V2V & vehicle-to-vehicle & VL & Visible Light \\
\hline WPT & Wireless Power Transfer & & \\
\hline
\end{tabular}

an emerging technology for green IoT that provides a single infrastructure for jointly sensing and transmitting data with micro-watt levels of power consumption as shown in Figure 2 , with the potential for enabling battery-free IoT devices. BackCom has been deployed for various applications such as food and liquid quality testing with Radio Frequency (RF) backscatter [13], communication with battery-free mobile phones [14], backscatter-assisted vehicular networks [15], underwater monitoring with acoustic backscatter [16] and gesture sensing with visible light backscatter [17].

There exist several surveys [18], [19], [20], [21] and [22] geared towards green communication and sensing in IoT. Authors in [18] presented an extensive survey of joint RF communications and sensing applications with the purpose of providing a platform for developing systems that would address the sensing and communications bottleneck in RF spectrum usage jointly rather than individually. Application scenarios for joint communications and sensing such as radar, vehicle-to-vehicle (V2V) communication, LiDAR, LiFi, asset tracking and radio frequency identification (RFID) were discussed. The authors also defined various topologies for the two-user scenario in RF spectrum access, including joint multiple access, monostatic broadcast channel, bistatic broadcast channel and in-band full duplex topology. Further, the authors explained various levels of integration between the sensing and communication systems such as non-integration, coexistence, cooperation and co-design in the joint two-user applications. Lastly, state-of-the-art techniques such as spread spectrum, orthogonal frequency division multiplexing (OFDM), adaptive modulation and multiple-input multiple-output (MIMO) reconfigurable systems and joint coding and challenges to joint communications and sensing were discussed. Authors in [19] discussed ambient BackCom (AmBC) as a paradigm for enabling green IoT. AmBC involves reflecting ambient wireless signals such as $\mathrm{WiFi}$, TV and cellular from a source using a sensor or tag to transmit information to a receiver. Due to the low power required by the AmBC sensors, they rely on harvested energy from ambient sources rather than batteries for operation. This allow for an easy to deploy, low cost, spectrum efficient and battery-free implementation of IoT. The authors described the architecture of $\mathrm{AmBC}$, its operating principles and advantages. They also identified and discussed challenges including allocation of harvested energy from the source, channel capacity, channel estimation, multiple access, interference at the receiver and security. Authors in [20] addressed the sustainability of large scale IoT deployment by providing a comprehensive discussion on EH-IoT. The authors discussed the commercialization and standardization activities related to IoT products and services that have taken place. Since IoT involves sensing, computing and communication, the authors comprehensively discussed EH techniques and their challenges in each of these areas. For sensing, EH process was discussed as a proxy for context sensing. Contextual sensing using EH patterns from various sources such as kinetic, thermoelectric, solar, and RF power were discussed. For computing, efficient check pointing and time scheduling of intermittently powered EH-IoTs were discussed. For communication, novel techniques including reinforcement learning (RL) assisted EHIoT, packetless communication and reflective radio with RFID, AmBC and intelligent reflective surfaces (IRS) were discussed. Authors in [21] provided a survey of green communication and sensing techniques designed for sustainable IoT systems. A critical consideration while discussing those techniques is ensuring that quality of service (QoS) is not compromised at the expense of energy efficiency. The green sensing techniques discussed include sensor duty cycling, wake-up radio, sensor scheduling and adaptive sampling. On the other hand, the green communication techniques discussed include data pruning by compression and by prediction. In addition to greening sensing and communication, authors also discussed EH techniques that can further sustain IoT devices by storing the harvested energy in capacitors, super capacitors or rechargeable batteries. The challenges and future research issues of green sensing and communication were also discussed. Lastly, authors discussed green sensing and communication in some application scenarios such as smart metering, smart grid health monitoring and lab environment monitoring. Authors in [22] presented a survey of energy efficient techniques for 
Internet of Wearable Things (IoWT). Since IoWT employs battery powered devices with small form factors, which need secure on-board computation, it requires more stringent energy management techniques. The authors categorized IoWT into healthcare, activity recognition, smart environments and general applications. Further, merits, demerits and performance metrics in each category of application were identified. Stateof-the-art energy efficient (EE) techniques were discussed, and future research works related to IoWT were highlighted.

Despite the breadth of existing surveys, they do not provide a comprehensive discussion on BackCom as a promising technique for greening IoT through joint sensing and communication. Strengths and gaps in existing studies are shown in Table II. Hence, this paper aims to fill these gaps by making the following contributions;

1) Describe the operation of joint sensing and communication with IoT, its evolution and merits over existing green IoT techniques;

2) Discuss state-of-the-art applications of BackCom green IoT;

3) Discuss techniques for greening BackCom;

4) Discuss challenges to adoption, possible solutions and future applications of BackCom green IoT.

The paper is structured as follows. The current section gives an introduction to the issue of green IoT. Section II describes the operation principle of BackCom based green IoT, and its evolution and architecture. Then Section III describes state-of-the-art implementations of green IoT with BackCom. Techniques for energy efficient operation of BackCom are presented in Section IV. Section V describes some challenges faced by BackCom based green IoT and proffers solutions to these challenges. Section VI discusses some possible future applications of BackCom based green IoT. Lastly, Section VII concludes the paper.

\section{GReen Wireless Communication And Sensing WITH BACKSCATTER COMMUNICATION}

This section gives a description of BackCom, its categories and the architecture of BackCom-based IoT. Also, its merits over conventional green IoT techniques and the evolution of joint sensing and communication applications with BackCom are discussed.

\section{A. Description of BackCom, Types of BackCom and Architec- ture of BackCom-based Green IoT}

In BackCom, sensed data at a sensor (tag) are modulated unto an incident carrier signal and reflected to a receiver. The carrier signal could be from WiFi, RFID, acoustic, frequency modulation (FM) radio, cellular or visible light source (exciter). The receiver could be collocated with the signal source or another device with computational capabilities. In essence, BackCom sensors transmit information by reflecting signals from other sources rather than employing power-hungry transmitters to generate carrier signals, modulate them with the data and amplify them for emission. This enables sustainable battery-free IoT devices that can sense and transmit data while operating at the micro-watt levels. The modulation technique

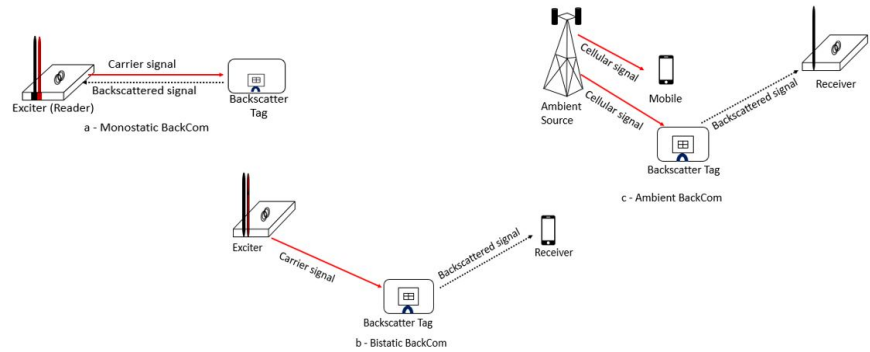

Figure 3: Illustration of Monostatic, Bistatic and Ambient BackCom

employed at the sensor could be analog or digital depending on the type of sensed data. For instance; a BackCom-based remote control could encode "ON" and "OFF" state data as "1" and " 0 ". An incident signal $\left(\mathrm{S}_{\mathrm{s}}\right)$ from a source can be modulated using instantaneous values of the reflection coefficient of the tag's antenna $\left(\tau_{\mathrm{i}}\right)$ to obtain a reflected signal $\left(\mathrm{S}_{\mathrm{r}}\right)$.

$$
S_{\mathrm{r}}=\tau_{\mathrm{i}} \cdot S_{\mathrm{s}}
$$

In order to vary the values $(0,1)$ of reflection coefficient $\tau_{\mathrm{i}}$, a mismatch is created between the tag's antenna impedance $Z_{i}$ and the load impedance $Z_{0}$

$$
\tau_{\mathrm{i}}=\frac{Z_{\mathrm{i}}-Z_{0}}{Z_{\mathrm{i}}+Z_{0}}
$$

BackCom systems can be categorized based on their excitation source into monostatic, bistatic and ambient BackCom. In monostatic BackCom [23]-[25], a dedicated exciter (reader) sends a carrier signal whenever it wants to communicate with the tag. The tag then reflects the carrier signal back to the reader. Hence, the reader acts as a transceiver. With monostatic BackCom, there is always a stable and high energy carrier signal for energy harvesting and reflection at the tag back to the receiver (reader), and it is easy to deploy for sensing and communication applications. In bistatic BackCom [26], [27], a distributed source (distributed exciter and receiver) is adopted. This means the tag could be brought closer to an exciter, thereby enhancing the operating range of the tags. In AmBC, a carrier signal originally meant for other communication purposes (such as FM radio [28], TV or WiFi [29]) is exploited to achieve BackCom. AmBC significantly reduces the cost of sensing and communication since it does not require a dedicated source. However, ambient communication signals are not always available for exciting the backscatter tags. Sometimes, they may be very weak due to fading or shadowing. Hence, utilizing such signals require careful design of tags. The three categories of BackCom are illustrated in Figure 3, while some applications of each category in IoT are shown in Table III

The architecture of BackCom-based green IoT system includes a signal source that provides an incident signal at the BackCom sensor (tag), the tag itself, and a receiver. At the tag, the incident signal serves as a carrier signal and an energy source for $\mathrm{EH}$ and powering the operation of the tag. The harvested energy is stored in a capacitor or small rechargeable battery. In addition to harvesting energy from the incident signal, energy could be harvested from solar energy, kinetic energy or other available ambient sources of energy. 
Table II: Strengths and identified gaps in existing studies

\begin{tabular}{|c|c|c|c|}
\hline Ref & Year & Strength(s) & Gap(s) in study \\
\hline$\overline{[18]}$ & $\overline{2016}$ & $\begin{array}{l}\text { The notion of joint RF spectrum utili- } \\
\text { zation for communication and sensing } \\
\text { was clearly explained. In relation to } \\
\text { two-user scenario,different topologies, } \\
\text { levels of integration and state-of- } \\
\text { the-art implementations and challenges } \\
\text { were discussed extensively. }\end{array}$ & $\begin{array}{l}\text { The discussion of joint communication } \\
\text { and sensing was limited to RF systems. } \\
\text { Though the study discussed co-design } \\
\text { of converged sensing and communication, } \\
\text { but it considered two independent infra- } \\
\text { structures that are co-designed. What } \\
\text { of a single energy efficient infra- } \\
\text { structure for both operations? }\end{array}$ \\
\hline [19] & 2019 & $\begin{array}{l}\text { AmBC was clearly discussed. Its } \\
\text { architecture, working principle } \\
\text { and advantages were highlighted. } \\
\text { Despite the challenges discussed } \\
\text { in the study, its potential for } \\
\text { greening IoT was proved. }\end{array}$ & $\begin{array}{l}\text { There exist other single } \\
\text { infrastructure systems for joint } \\
\text { sensing and communications } \\
\text { beside AmBC that were not } \\
\text { discussed. They include; } \\
\text { monostatic and bistatic } \\
\text { architecture backscatter } \\
\text { communications }\end{array}$ \\
\hline [20] & 2019 & $\begin{array}{l}\text { EH-IoTs and novel techniques } \\
\text { for ensuring sustainable operation } \\
\text { of sensing, computation and } \\
\text { communication were } \\
\text { comprehensively discussed }\end{array}$ & $\begin{array}{c}\text { Despite comprehensively } \\
\text { discussing EH-IoTs, BackCom } \\
\text { was only discussed as a technique } \\
\text { to enhance communication. } \\
\text { BackCom's potential for joint } \\
\text { sensing and communication was } \\
\text { not discussed comprehensively }\end{array}$ \\
\hline$[21]$ & 2020 & $\begin{array}{l}\text { The authors discussed green } \\
\text { techniques for sensing and } \\
\text { communication applications } \\
\text { without compromising QoS in } \\
\text { those applications. EH and storage } \\
\text { techniques in IoT were discussed. }\end{array}$ & $\begin{array}{l}\text { The authors discussed green } \\
\text { sensing and communication } \\
\text { techniques independently. Though } \\
\text { EH was discussed, BackCom as a } \\
\text { green technique for joint } \\
\text { communication and sensing was } \\
\text { not discussed. }\end{array}$ \\
\hline [22] & 2020 & $\begin{array}{l}\text { The authors comprehensively } \\
\text { discussed existing studies on EE } \\
\text { IoT and classified various } \\
\text { IoWT into categories. Highlighted } \\
\text { advantages, disadvantages and } \\
\text { performance metrics of each } \\
\text { category }\end{array}$ & $\begin{array}{l}\text { The discussion of EE techniques } \\
\text { is limited to wearable IoT. Also, } \\
\text { a discussion on joint sensing and } \\
\text { communication with BackCom is } \\
\text { not presented }\end{array}$ \\
\hline
\end{tabular}

Table III: Applications of different categories of BackCom in IoT

\begin{tabular}{|c|c|c|c|}
\hline \hline Category & Application(s) & Advantage(s) & Disadvantage(s) \\
\hline \hline Monostatic & $\begin{array}{c}\text { Massive IoT [30], Multi-antenna } \\
\text { RFID [31], Multi-tag RFID [32] }\end{array}$ & Ease of deployment & High cost of reader \\
\hline Bistatic & $\begin{array}{c}\text { Extended IoT operation } \\
\text { range [33], [34] }\end{array}$ & Enhanced range & $\begin{array}{c}\text { Interference at the receiver } \\
\text { between tag and reader }\end{array}$ \\
\hline Ambient & $\begin{array}{c}\text { Mobile edge computing [12], } \\
\text { Indoor localization [35], } \\
\text { Distributed IoT network [36] }\end{array}$ & $\begin{array}{c}\text { Low cost of } \\
\text { deployment }\end{array}$ & $\begin{array}{c}\text { Non-stability of ambient } \\
\text { signals }\end{array}$ \\
\hline
\end{tabular}

The harvested energy is used to power a switch that can change between various states of load depending on the value of the sensed signal. Also, the switching mechanism is combined with the antenna to modulate the sensed data unto the incident carrier signal. BackCom based green IoT has shown good energy saving and network performance [37]. The architecture of joint sensing and communication with BackCom is shown in Figure 4. Also, a comparison between conventional green IoT techniques and BackCom green IoT is presented in Table IV.

\section{B. Evolution of Joint Sensing and Communication with Back- Com}

Joint sensing and communication with BackCom has a long history that started in around 1945, when a spying device called the Great Seal bug [38] was surreptitiously placed in the US Embassy in the Soviet Union to eavesdrop on conversations by modulating the acoustic (speech) signals unto a high frequency carrier signal from a transmitter placed

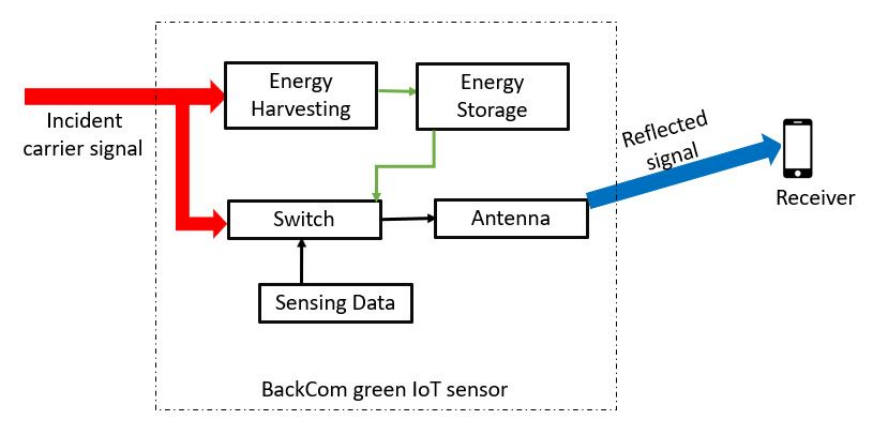

Figure 4: Architecture of BackCom-based Green IoT 
Table IV: Comparison between conventional green IoT techniques and BackCom-based green IoT

\begin{tabular}{|c||c|}
\hline \hline Coventional green IoT & BackCom-based green IoT \\
\hline \hline $\begin{array}{c}\text { More energy consumption due to non- } \\
\text { elimination of power-hungry components } \\
\text { (such as; oscillators and power amplifiers) }\end{array}$ & $\begin{array}{c}\text { Less energy consumption due to elimination } \\
\text { of power-hungry components }\end{array}$ \\
\hline $\begin{array}{c}\text { Cannot guaranttee battery-free operation and } \\
\text { small form factor applications }\end{array}$ & $\begin{array}{c}\text { Can guaranty battery-free operation and } \\
\text { small form factor applications }\end{array}$ \\
\hline $\begin{array}{c}\text { Green techniques target a certain aspect of } \\
\text { the IoT node }\end{array}$ & $\begin{array}{c}\text { Jointly performs sensing and communication } \\
\text { at green budgets }\end{array}$ \\
\hline $\begin{array}{c}\text { Higher cost of deployment due to inability to } \\
\text { use other ambient excitation sources (such } \\
\text { as; WiFi and FM radio) }\end{array}$ & $\begin{array}{c}\text { Lower cost of deployment since they can use } \\
\text { ambient excitation sources (such as; WiFi } \\
\text { and FM radio) }\end{array}$ \\
\hline
\end{tabular}

Table V: Evolution of joint sensing and communication with BackCom

\begin{tabular}{|c|c|c|}
\hline \hline Reference & Year & Contribution \\
\hline \hline$[38]$ & $\mathbf{1 9 4 5}$ & Great Seal Bug \\
\hline$[39]$ & $\mathbf{1 9 4 8}$ & Feasibility of sensing and communication using reflected power \\
\hline$[40]$ & $\mathbf{1 9 6 3}$ & Passive RFID transponder \\
\hline$[26]$ & $\mathbf{2 0 1 2}$ & Bistatic architecture for BackCom \\
\hline$[29]$ & $\mathbf{2 0 1 3}$ & Ambient BackCom \\
\hline$[41]$ & $\mathbf{2 0 1 5}$ & VL BackCom \\
\hline$[42]$ & $\mathbf{2 0 1 7}$ & Electronics-free IoT with BackCom \\
\hline$[43]$ & $\mathbf{2 0 1 8}$ & Securing BackCom-based IoT using propagation signature \\
\hline$[44]$ & $\mathbf{2 0 1 9}$ & Sensing underwater environment with acoustic BackCom IoT \\
\hline$[45]$ & $\mathbf{2 0 2 0}$ & Hybrid (RF and VL) based BackCom IoT \\
\hline
\end{tabular}

outside the embassy and receiving the reflected modulated signals outside the embassy for decoding. In 1948, researchers reported a design and feasibility tests [39] on using reflected signal power for sensing and communication. In the experiment, a carrier signal was generated by a transmitter while the active radio transceiver was replaced by a modulated reflector. Subsequently, passive RFID transponders [40] were developed, commercialized and standardized. Initial deployment scenarios include toll gate fee collection, securing access to locations and equipment, e-passport, automobile, and management of logistics. Further, a distributed (bistatic) architecture aimed at enhancing the range of BackCom without relocating the excitation source was designed [26]. Then, AmBC was designed in [29] where ambient signal sources (TV and cellular) were used as sources of excitation thereby cutting the cost of deploying expensive readers as excitation sources. In order to push the evolution from visible light perspective, ambient visible light (VL) based BackCom was designed in [41]. Despite green operation of BackCom, authors in [42] designed 3D printed devices that can interact with one another by leveraging electronics-free BackCom. This work truly showed the potential of BackCom to enable Internet of Everything (IoE). Further, attempts to secure BackCom based IoT systems started with work in [43]. Then, the first implementation of sensing and communication with acoustic BackCom to enable underwater sensing and networking was reported in [44]. Also, a hybrid (RF and VL) BackCom [45] was developed to address the uplink and downlink challenges, respectively, of RF and VL BackCom. These existing works underscore the ability of BackCom-based IoT systems to perform joint sensing and communication simultaneously while maintaining green power budgets.

\section{STATE-OF-THE-ART APPLiCATIONS OF BACKCOM IOT}

This section gives a classification of applications of BackCom-based green IoT, and discusses state-of-the-art in each of the applications.

\section{A. Healthcare}

A BackCom based green IoT system was presented in [46] for monitoring physical parameters on a human body. The green BackCom sensors allowed the body area network of IoT devices to transmit the sensed parameters to commercial offthe-shelf (COTS) devices. Similarly, authors in [47] designed a system (wrist-worn band) for continuously monitoring human vitals in a hospital setting. Efforts to enable in-body health monitoring were presented in [48], [49]. These systems were able to address problems of charging and inaccurate implant localization respectively by leveraging antenna with high power beams for wireless implant charging and solving in-body RF channel impairments.

\section{B. Human Activity Recognition (HAR)}

The earliest wearable device-free and battery-free continuous gesture recognition using BackCom was designed by the authors in [50], wherein experiments using ambient RFID and TV signals were able to achieve high accuracy in identifying gestures. Authors in [51] leveraged the non-stability of ambient signals to perform gesture recognition. High accuracy was achieved even when the ambient signal strength is low. Further, authors in [52] designed a scalable green BackCom system for monitoring human activity using multiple BackCom tags. The channel state information (CSI) between tags was used to identify the various activities. Authors in [53] designed a BackCom based system that can trace human motion using the reflected signals from the BackCom sensor. This technique 
of motion tracing showed promising results in addressing occlusion suffered by camera-based motion tracing. Also, BackCom was leveraged to develop sentence level language recognition in order to enhance communication with deaf people and recognize speech affected by dysphonia [54], [55].

\section{Agriculture}

Authors in [56], [57] designed a bistatic BackCom-based green IoT for monitoring plant physiology by checking the plant's stress level. To make plant stress monitoring greener, authors in [58] harvested energy from plants being monitored to combine with harvested energy from incident RF signal. In [28], an AmBC-based green IoT underlaying FM radio signals was used to monitor plants by sensing the temperature difference between leaves and plants. To exploit diversity of ambient FM radio signals to achieve better tag performance, authors in [59] designed tags that can select the best incident FM radio signal for operation. Authors in [60], [61] designed green BackCom IoT techniques for monitoring plant physiology using moisture content levels.

\section{Transportation}

BackCom-based green IoT has been leveraged to enhance transportation systems. For instance, an automotive application based on BackCom [15] was developed in order to support interaction between vehicles and roadside infrastructure. The authors designed novel backscatter techniques based on VL BackCom. The techniques exploit the retro-reflective property of roadside signs to allow multiple access communication of dynamic information using the roadside signs. The techniques developed achieved a vehicle-to-infrastructure (V2I) communication range of $101 \mathrm{~m}$. Rather than leveraging $\mathrm{VL}$, the BackCom-enablde vehicular communication system in [62] uses RF signals from base stations (BS) connected to the vehicles together with roadside backscatter tags to ensure safety of vehicles while travelling. Authors in [63] designed an AmBC-assisted transportation system that uses simultaneous wireless information and power transfer (SWIPT) to enable communications between vehicles in a transportation network.

\section{E. Mobile IoT}

Building on pioneering work [42] that designed electronicsfree backscatter for IoT, authors in [64] designed printable passive tags that can sense human interaction. The tags leverage ambient signal variation based on the particular interaction with the tag to decode the sensed activity using mobile devices. Similarly, authors in [65] leveraged ambient FM radio signals to enable ubiquitous smart city applications. In the applications, custom billboards could leverage BackCom to communicate the messages they contain. Also, small tags embedded in fabrics were used for on-body sensing applications such as gait and vital signs monitoring. Lastly, authors in [66] leveraged Bluetooth based BackCom to design a robust and reliable low power communication technique for commodity IoT devices.

\section{TEChNiques FOR GREENING BACKCOM Systems}

This section explains various techniques that have been employed by BackCom systems to ensure operation of IoT at green budgets. The categories of techniques and works done under each category are explained.

\section{A. Energy Harvesting}

Green operation of BackCom-based IoT nodes require a constant supply of incident energy for $\mathrm{EH}$ at the tag or efficient power management techniques to avoid exhausting the harvested power by the tags. Performance analysis of combining EH and BackCom [67]-[69] has shown promise as a solution to these problems. Hence, authors in [70], [71] developed techniques to enable continuous availability of RF energy for harvesting at the tag. In [70], a dual band $(1.8 \mathrm{GHz}$ and $2.45 \mathrm{GHz}$ ) EH and backscatter circuit was presented. The circuit allows $1.8 \mathrm{GHz}$ signals to be used solely for $\mathrm{EH}$ while the other band is used for low power BackCom. Matching circuits designed for both bands ensures maximum power transfer to the sensor node for storage. In contrast, different bands at $5.83 \mathrm{GHz}$ and $3.45 \mathrm{GHz}$ were utilized in [71] for $\mathrm{EH}$ at the tag and low power BackCom, respectively. Additionally, the authors designed EE techniques for transmission of signals at $5.83 \mathrm{GHz}$. Further, authors in [72] designed a singleseries rectenna circuit with one double diode on a lossy FR-4 substrate for sensitive and efficient EH in BackCom. The losses incurred due to the substrate were compensated by ensuring suitable impedance matching network, rectifier microstrip trace dimensions and load were adopted. Simulated results showed that the rectenna could achieve $28.4 \% \mathrm{EH}$ efficiency for a $-20 \mathrm{dBm}$ incident signal.

Drawing from the benefits of multi-antenna systems, authors in [73] designed a wireless energy transfer (WET) technique for BackCom systems. A multi-antenna enabled energy transmitter (ET) with energy beamforming was leveraged to transfer energy to (and from) an energy receiver (ER) using the forward and backscatter channels. Since CSI is required for energy beamforming, the authors developed a channel estimation technique at the ET that utilizes the backscatter CSI rather than forward CSI because the BackCom sensor is limited powerwise. Results of the WET scheme proved its promise (10\% different from result with perfect forward CSI estimate) in powering wireless devices. Rather than leveraging energy beamforming for WET only, authors in [74] developed the first technique for joint energy and information transfer in BackCom. To achieve that, energy beamforming design was modeled as an optimization problem that seeks to maximize the minimum achievable data rate at the receiver subject to energy constraint. Results of the designed technique showed high $(90 \%)$ similarity to data rates achieved using BackCom receivers with perfect CSI.

Some BackCom applications are required to operate in long range scenarios, but long-range transmissions require a higher transmit power from the tags, which is a constrained resource. Therefore, authors in [75] designed a self-sustainable Long range Radio (LoRA)-enabled backscatter tag that operates at very low power $(240 \mathrm{nW})$ budget. The tag was designed with 
Table VI: Summary of energy harvesting techniques

\begin{tabular}{|c|c|c|c|}
\hline Reference & Aim & Implementation & Result \\
\hline [70] & $\begin{array}{l}\text { Ensure available RF source } \\
\text { for EH }\end{array}$ & $\begin{array}{c}\text { Design multiple RF band ( } 1.8 \text { and } \\
2.45 \mathrm{GHz}) \mathrm{EH} \text { circuit }\end{array}$ & $\begin{array}{l}\text { Increased harvested energy at } \\
\text { the tag }\end{array}$ \\
\hline [71] & $\begin{array}{c}\text { Ensure available RF source } \\
\text { for EH }\end{array}$ & $\begin{array}{c}\text { Design multiple RF band (3.45 and } \\
5.83 \mathrm{GHz}) \mathrm{EH} \text { circuit }\end{array}$ & $\begin{array}{l}\text { Increased harvested energy } \\
\text { the tag }\end{array}$ \\
\hline [72] & $\begin{array}{l}\text { Increase efficiency of EH } \\
\text { at the tag }\end{array}$ & $\begin{array}{l}\text { Design a single-series rectenna } \\
\text { circuit for EH }\end{array}$ & $\begin{array}{l}\text { High (24\%) EH efficiency even with } \\
\text { low }(-20 \mathrm{dBm}) \text { incident signal }\end{array}$ \\
\hline [73] & $\begin{array}{l}\text { EH for BackCom using } \\
\text { energy beamforming }\end{array}$ & $\begin{array}{l}\text { Leverage a multi-antenna ET for } \\
\text { energy transfer to tag }\end{array}$ & $\begin{array}{c}\text { High }(90 \%) \text { similarity in energy } \\
\text { transfer compared to receiver with } \\
\text { perfect CSI }\end{array}$ \\
\hline [74] & $\begin{array}{l}\text { EH for BackCom using } \\
\text { energy beamforming }\end{array}$ & $\begin{array}{l}\text { Model energy beamforming design } \\
\text { as max-min optimization problem }\end{array}$ & $\begin{array}{l}\text { High }(90 \%) \text { similarity in data rate } \\
\text { compared to receiver with perfect CSI }\end{array}$ \\
\hline [75] & $\begin{array}{c}\text { Achieve sustainable LoRA } \\
\text { BackCom }\end{array}$ & $\begin{array}{l}\text { Design highly sensitive EH circuit and } \\
\text { generate LoRA- like transmissions }\end{array}$ & $\begin{array}{l}\text { Harvested energy from RF signal } \\
\text { with low strength }(-22.5 \mathrm{dBm}) \text { and } \\
\text { long range }(381 \mathrm{~m}) \text { transmission }\end{array}$ \\
\hline [76] & Achieve LoRA BackCom & $\begin{array}{l}\text { Enable EH from two-way radio carrier } \\
\text { and use the carrier for BackCom } \\
\text { random channel }\end{array}$ & $\begin{array}{l}\text { More EE operation of } \\
\text { AmBC network }\end{array}$ \\
\hline [77] & $\begin{array}{l}\text { Enhance coverage of } \\
\text { multi-tag BackCom }\end{array}$ & $\begin{array}{l}\text { Design algorithm to mitigate phase } \\
\text { cancellation in tag-to-tag BackCom }\end{array}$ & $\begin{array}{l}\text { Enabled tag-to-tag transmissions in } \\
\text { BackCom with RF EH }\end{array}$ \\
\hline
\end{tabular}

highly sensitive RF energy harvesting and low power consuming circuit to maximize EH. During backscatter operation, LoRa-like transmissions at the tag were achieved by direct digital frequency synthesis at the tag. The designed tag could harvest energy from very low strength $(-22.5 \mathrm{dBm})$ signals and transmit over a long $(381 \mathrm{~m})$ distance. In contrast to harvesting energy from a dedicated RF transmitter, authors in [76] designed an on-body long range wearable BackCom device that harvests energy from a two-way radio operating at 464.5 MHz . A sensing backscatter tag and an on-body device harvest energy from the $464.5 \mathrm{MHz}$ signal independently. In addition to harvesting RF energy, the on-body device uses the signal from the two-way radio to generate a second harmonic signal that is used for interrogating the backscatter tag. This allows for EE operation in AmBC with single and multiple tags compared to conventional AmBC.

In multiple tag BackCom, tag-to-tag communication can be leveraged to enhance the range and coverage of BackCom operation. However, adopting tag-to-tag communication with amplitude shift keying (ASK) modulated carrier signal leads to phase cancellations during decoding process (using envelope detector) at the tag. Hence, authors in [77] developed a phase cancellation mitigation algorithm based on phase rotation control to ensure efficient operation of BackCom systems with RF EH.

\section{B. Security}

Low power budgets of BackCom systems make them vulnerable to security threats such as information leakage through covert channels [78], [79] due to non-adoption of power-consuming protocols with highly secure encryption. To demonstrate the vulnerability of BackCom, authors in [78] showed how information can be leaked from mobile devices using backscatter based covert channels. The system exploits the changing impedance of WiFi network interface card (NIC) due to different working states with few/no extra software commands to establish the covert channels. The covert channels could achieve $1.6 \mathrm{bps}$ throughput over $2 \mathrm{~m}$ range. Hence, the porous nature of the BackCom physical layer poses security threat. Another threat to security of BackCom is jamming attack from signals with higher strength. In order to secure the physical layer, authors in [80] leveraged backscatter tags attached to an access point (AP) in a multi-antenna like structure to secure IoT devices. The structure at the AP allows for fine-grained multipath signature assignment to legal user and an attacker. When interacting with a user, the AP uses dynamic time warping (DTW) and support vector machines (SVM) to identify and prevent attacks. Similarly, authors in [43] used the propagation signature of on-body signals to secure backscatter networks. Since the signals refracted due to body tissues are different from off-body signals, attacks could be easily identified. The challenge of separating on-body propagation signatures from noise (caused by body movement) is addressed by comparing the backscatter signal with a direct path on-body signal. Another physical layer solution based on leveraging random artificial noise at the tag was presented in [81]. The system randomly generates a noise signal at the tag and transmits it to a user and an eavesdropper. This results in receiving a backscatter signal corrupted with noise. The receiver then applies successive interference cancellation (SIC) to eliminate the noise.

For securing RFID based sensing systems, authors in [82] leveraged randomization to secure RFID tags without adding any components. The designed RFID system randomizes modulation by generating a random carrier at the reader every time it wants to sense the tag. This leads to a random modulation. Then, the wireless channel for backscatter is also randomized by randomly switching between moving antennas at the tag. The RFID system could defend against MIMO and nonMIMO attackers. Rather than randomizing both the channel and modulation, authors in [83] only randomized the carrier wave $(\mathrm{CW})$ generated by the multi-antenna RFID reader. Since the reader has prior knowledge of the variation in the $\mathrm{CW}$, it is able to decode the backscatter signal while adding secrecy to the eavesdropper. Further, a two-factor authentication was proposed in [84] to secure RFID tags. The first authentication factor is generated by making a unique tapping on the tag by the user. This causes unique phase changes to the backscattered signals which are decoded by the reader. For the second factor, the reader compares the extracted sequence and compares it with the stored tapping sequence of the user. If both factors are okay, then the user passes the authentication.

Since a jamming attack prevents transmission of signals, a backscatter device can utilize the jamming signal as a carrier to modulate information, thereby continuously harvesting energy and communicating with a receiver. Hence, The authors in [85] designed a technique to defend against jamming attack 
Table VII: Summary of security techniques

\begin{tabular}{|c|c|c|c|}
\hline Reference & Aim & Implementation & Result \\
\hline [80] & $\begin{array}{l}\text { Secure AP with multi-antenna } \\
\text { structure }\end{array}$ & $\begin{array}{l}\text { Assign fine grained signatures to legal and } \\
\text { attacking devices at the AP using tags }\end{array}$ & $\begin{array}{c}\text { Accurately detect } 97 \% \text { of } \\
\text { attacks }\end{array}$ \\
\hline [43] & $\begin{array}{l}\text { Exploit on-body signal propagation } \\
\text { to secure backscatter networks }\end{array}$ & $\begin{array}{l}\text { Develop algorithm to separate between } \\
\text { on-body and off-body signal in the } \\
\text { presence of noise }\end{array}$ & $\begin{array}{l}\text { Achieved } 93.2 \% \text { true positives } \\
\text { and } 3.18 \% \text { false positives }\end{array}$ \\
\hline [81] & $\begin{array}{l}\text { Secure backscatter tags with } \\
\text { random artificial noise }\end{array}$ & $\begin{array}{l}\text { Generate noise and transmit with } \\
\text { backscatter signal. And use SIC at } \\
\text { legal receiver }\end{array}$ & $\begin{array}{l}\text { Achieved high secrecy rate which } \\
\text { increases with increase in tag-reader } \\
\text { channel gain and number of tags }\end{array}$ \\
\hline [82] & $\begin{array}{l}\text { Secure RFID systems with } \\
\text { randomization }\end{array}$ & $\begin{array}{l}\text { Create random carrier for modulation } \\
\text { and randomly switch antenna to create } \\
\text { random channel }\end{array}$ & $\begin{array}{l}\text { Defend against MIMO and } \\
\text { non-MIMO attackers }\end{array}$ \\
\hline [83] & $\begin{array}{l}\text { Secure RFID systems with } \\
\text { randomization }\end{array}$ & Create random variations in the $C W$ & Increase secrecy of AmBC network \\
\hline [84] & $\begin{array}{l}\text { Two-factor RFID tag } \\
\text { security }\end{array}$ & $\begin{array}{l}\text { Randomly create unique phase changes at } \\
\text { the tag. Authenticate using saved } \\
\text { sequence at the reader. }\end{array}$ & $\begin{array}{l}\text { Achieved near zero false positives } \\
\text { and false negatives }\end{array}$ \\
\hline [85], [86] & $\begin{array}{l}\text { Defend against jamming attacks } \\
\text { on devices with backscatter }\end{array}$ & $\begin{array}{l}\text { Utilize the high tone jamming signal as } \\
\text { a carrier to communicate with receiver }\end{array}$ & $\begin{array}{l}\text { Continuous energy harvesting and } \\
\text { transmission by jammed devices }\end{array}$ \\
\hline [87] & Defend against smart jammers & $\begin{array}{l}\text { Deceive the jammer by using } \\
\text { false transmission }\end{array}$ & $\begin{array}{l}\text { Defend against jamming and } \\
\text { improve performance }\end{array}$ \\
\hline [88] & Defend against smart jammers & $\begin{array}{l}\text { Learn the operation of jammer and } \\
\text { use it to adapt BackCom }\end{array}$ & $\begin{array}{l}\text { Improved throughput }(426 \%) \text { and lower } \\
(24 \%) \text { packet loss rate }\end{array}$ \\
\hline [89] & $\begin{array}{l}\text { Defend against jamming in a } \\
\text { multi-tag AmBC network }\end{array}$ & $\begin{array}{l}\text { Leverage artificial noise from } \\
\text { tags and tag cooperation }\end{array}$ & $\begin{array}{l}\text { Improved BER and secrecy rate } \\
\text { of AmBC network }\end{array}$ \\
\hline
\end{tabular}

by utilizing the jamming signal. To address the uncertainty of using the jamming signal by the transmitter, an RL approach is used to attain an optimal policy. To leverage strong feature extraction of deep learning, authors in [86] developed a deep reinforcement learning (DRL) technique in order to obtain a more optimal policy to prevent jamming attacks. Some jammers are smart in a way that they only attack BackCom channel when a legitimate transmitter is operating. To prevent such smart jamming attacks, authors in [87] developed intelligent techniques that deceive the jammers by transmitting fake signals from legitimate devices. When the jammers attack, the jamming signal is used for backscatter or EH. Rather than deceiving the smart jammers, authors in [88] leveraged deep neural networks to first learn operation of the smart jammer. Then, the AmBC device adapts its operation parameters (such as transmit power and data rate) based on the operation of the jammer to avoid attacks.

For BackCom networks with multiple tags, authors in [89] developed security techniques based on artificial noise generation and tag cooperation to defend against jamming attacks. In the former, the BackCom network selects some tags to randomly generate noise and transmit together with the valid packet, thereby making the eavesdropper experience interference. In the latter, the tags cooperate to transmit the same information in order to assist decoding at the receiver in case of packet loss from some of the tags.

\section{Channel Modeling and Measurement}

The channel of BackCom-based IoT requires careful modelling due to its extra complexity over conventional communication channels. With BackCom-based IoT opening up some application scenarios that are not previously addressed with conventional IoT, additional modelling parameters need to be considered. A discussion on some of the channel models and related empirical studies are presented below.

Since BackCom involves modulating data at the tag by creating impedance mismatch, a key factor in enhancing the performance of BackCom is the relationship between incident signal and tag impedance. Hence, authors in [90] proposed a real-time impedance characterization technique for RFIDtype BackCom with low cost devices such as oscilloscope, computer and signal generator. The setup obtained near accurate impedance measurement during the tag's operation under a varying $\mathrm{CW}$, hence providing insight on how to optimize the tag design. However, ambient radio signals (e.g., WiFi, cellular and FM radio signals) often already have modulated information. Hence the range of backscatter signalling will have certain limitations. To analyse those limitations, an RF link budget that models the expected returns and range was developed in [91] and [92] for FM radio and WiFi based AmBC, respectively. Further, the authors in [93] modelled retro-directive tags' backscatter channel in the presence of fading. The retro-directive channels showed fading behaviours similar to one-way radio links, and the channels were highly sensitive to the structure of the diffused multipath waves.

Agricultural BackCom-based IoT systems promised a low cost means of monitoring plants [57] [61]. However, existing propagation models do not account for the effects of grass moisture on near-ground RF propagation in a large nonuniform grass area. Authors in [94] conducted measurements of signal strengths between two horn antennas in a nearground setting with varying moisture on grasses at 3-4 GHz. In the experiments, decreasing the moisture content of the grasses caused decreasing signal strengths, implying a dielectric waveguide effect as a result of wet grasses. These experiments also demonstrated the promise of using propagation characteristics to sense the moisture contents in vegetation.

Despite the low cost of $\mathrm{AmBC}$, it is the most complex category of BackCom in terms of model development. Hence, authors in [95] developed mathematical models for the legacy link and backscatter link. Then, various receiver architectures were used to analyze the bit error rate (BER) and interference of the AmBC network. Simulation of the system showed the receiver could suppress the interference from the legacy link at the receiver. Similarly, authors in [96] modelled the channel of AmBC-based IoT using cellular network signals as ambient carrier. In contrast to existing models, authors in [96] considered the effects of interference between legacy transmissions and backscatter transmission, interference between backscatter nodes, and non-linear EH model in modelling and analysing the backscatter system. A time-switching (TS)/power splitting (PS) operation scheme was designed where devices can harvest 
Table VIII: Summary of channel modeling and measurement techniques

\begin{tabular}{|c|c|c|c|}
\hline Reference & Aim & Implementation & Result \\
\hline$[90]$ & $\begin{array}{c}\text { Investigate the behaviour of tag } \\
\text { impedance with varying CW }\end{array}$ & $\begin{array}{c}\text { Use low-cost devices to obtain } \\
\text { real-time impedance values }\end{array}$ & $\begin{array}{c}\text { Measured impedance was used } \\
\text { to optimize tag design }\end{array}$ \\
\hline$[91],[92]$ & $\begin{array}{c}\text { Investigate the expected returns } \\
\text { and gains of backscatter channel }\end{array}$ & $\begin{array}{c}\text { Model the backscatter channel } \\
\text { considering the mechanism of } \\
\text { RF propagation }\end{array}$ & $\begin{array}{c}\text { A complete BackCom link budget was } \\
\text { developed for system characterization }\end{array}$ \\
\hline$[93]$ & $\begin{array}{c}\text { Investigate the expected returns } \\
\text { and gains of backscatter channel }\end{array}$ & $\begin{array}{c}\text { Model the channel of retro-directive } \\
\text { tags in the presence of fading }\end{array}$ & $\begin{array}{c}\text { Retro-directive channels showed similar } \\
\text { properties to one-way radio link }\end{array}$ \\
\hline$[94]$ & $\begin{array}{c}\text { Investigate the effects of } \mathbf{R F} \\
\text { propagation in wet grasses }\end{array}$ & $\begin{array}{c}\text { Take signal strength measurements } \\
\text { between two horn antennas } \\
\text { close to the ground }\end{array}$ & $\begin{array}{c}\text { Moisture showed direct relation with } \\
\text { signal strength implying } \\
\text { waveguide effect }\end{array}$ \\
\hline$[95]$ & $\begin{array}{c}\text { Model and analyze AmBC } \\
\text { network }\end{array}$ & $\begin{array}{c}\text { Develop models for legacy and } \\
\text { backscatter link and analyze } \\
\text { capacity bounds }\end{array}$ & $\begin{array}{c}\text { Legacy link interference is } \\
\text { addressed using OFDM. }\end{array}$ \\
\hline$[96]$ & $\begin{array}{c}\text { Investigate effect of interference } \\
\text { in BackCom channel underlaying } \\
\text { cellular network }\end{array}$ & $\begin{array}{c}\text { Model an operating scheme that } \\
\text { switches between harvesting and } \\
\text { transmitting times }\end{array}$ & $\begin{array}{c}\text { Near optimal outage capacity } \\
\text { was achieved }\end{array}$ \\
\hline$[97]$ & $\begin{array}{c}\text { Investigate city-wide ambient } \\
\text { RF signal density }\end{array}$ & $\begin{array}{c}\text { Measure RF power levels along roads } \\
\text { and busy areas with human traffic }\end{array}$ & $\begin{array}{c}\text { Cellular communication bands were } \\
\text { most suitable for BackCom in cities } \\
\text { Estimate channel of multi-tag } \\
\text { (up to five) AmBC network. }\end{array}$ \\
\hline$[98]$ & $\begin{array}{c}\text { Channel estimation in multi-tag } \\
\text { AmBC }\end{array}$ & $\begin{array}{c}\text { Model the relationship between moving } \\
\text { tag signals and collision }\end{array}$ & \\
\hline & & &
\end{tabular}

energy, modulate and reflect ambient signals. Numerical results of TS/PS scheme showed a near optimal outage capacity performance. While previous simulation and numerical studies showed the theoretical performance limits of AmBC, authors in [97] conducted ambient RF density measurements for citywide planning of BackCom-based IoT network deployment. The RF power level measurements were conducted along major roads, highways and high traffic density areas of Montreal in the $400-2700 \mathrm{MHz}$ frequency spectrum. Cellular bands showed higher suitability (in urban areas) while TV bands showed higher suitability (in suburban areas) for AmBC.

In multiple-tag BackCom networks, performance of sensing applications is limited due to difficulty in estimating the parallel channels between the tags and the receiver. Authors in [98] proposed a novel technique for channel estimation in multitag AmBC. Based on the insight that movement of signals in the presence of collision is preserved despite variation in tag modulation, models for characterizing the relationship between signal variation and collision were used for channel estimation. The proposed technique enabled channel estimation of a 5-tag AmBC network with high accuracy.

\section{Multiple Access}

Communication between a transmitter and a receiver requires techniques that allow the transmitter to receive feedback from the receiver. This helps in addressing challenges such as packet collision, rate adaptation and data re-transmission. Pioneering work by authors in [99] leveraged BackCom to design a full duplex feedback channel. The receiver uses a simple circuit with analog components to instantaneously reflect the received signal back to the transmitter without disrupting the transfer of information from the transmitter. The receiver circuit consumed a very low $(0.54 \mu \mathrm{W})$ power. Building on this pioneering work, authors in [100], [101] enhanced full duplex communication in BackCom. In [100] a novel multiple access scheme based on time hopping spread spectrum (THSS) was designed to enable full duplex information transfer between BackCom devices. The scheme involves allowing each tag to modulate data unto the incident carrier using binary phase shift keying (BPSK) in its corresponding time hop. Then, a reader is used to coherently decode the backscattered bits. On the other hand, authors in [101] developed a full duplex access point (FAP) that can simultaneously transmit ambient RF signal to legacy users and also decode received signals from backscatter devices. Resources for each task are allocated while ensuring system throughput maximization and fairness.

Rather than developing multiple access techniques at transmitter or receiver, authors in [102] designed a novel channel model to allow multiple access AmBC. They proposed the multiplicative multiple access channel (M-MAC) that considers the received backscatter channel signal as a multiplication of RF source signal and the tag's signal. The scheme allows detection of both direct link ambient RF signal and backscatter signal simultaneously at the receiver rather than treating the direct link RF signal as interference. Analysis of achievable rates of M-MAC showed throughput increase over conventional BackCom channel.

Though many conventional ambient RF sources such as WiFi have adopted OFDMA carriers, analysis of BackCom with non-orthogonal multiple access (NOMA) [107], [108] has shown the potential of improving existing and future BackCom-based IoT. Hence, authors in [103] leveraged NOMA for greening BackCom through enhanced spectrum efficiency. The authors proposed a hybrid time division multiplexing access (TDMA) and power domain-NOMA (PDNOMA) to enable multiple sensors access the uplink (tagto-reader) channel. Experiments with the proposed scheme showed better throughput over conventional TDMA. Rather than leveraging NOMA, authors in [104] developed a scalable uplink multiple access protocol based on slotted ALOHA. The protocol allows the reader to estimate the number of tags in the network and assign a slot to each tag. During the operation of the network, the reader adapts the number of tags based on the packets received. Results of the developed protocol showed multiple tag access with less collisions. Further, authors in [105] designed a technique for efficient resource allocation in NOMA enhanced BackCom. The resource allocation scheme was modelled as a convex optimization problem aimed at maximizing the minimum throughput of backscatter devices. This was achieved by jointly optimizing BackCom devices' backscatter time and power reflection coefficient with constraints on harvested energy at the tag, signal-to-interferencenoise-ratio (SINR) and other practical parameters. Compared to orthogonal multiple access (OMA) with traditional TDMA, the proposed technique showed significant throughput gain. Despite existing techniques for multiple access showing EE 
Table IX: Summary of multiple access techniques

\begin{tabular}{|c|c|c|c|}
\hline Reference & Aim & Implementation & Result \\
\hline$[100]$ & Enable full duplex BackCom & $\begin{array}{c}\text { Design multiple access based } \\
\text { on THSS }\end{array}$ & $\begin{array}{c}\text { Achieved full duplex transmission } \\
\text { at the receiver }\end{array}$ \\
\hline$[101]$ & Enable full duplex BackCom & $\begin{array}{c}\text { Design an AP for simultaneous uplink } \\
\text { and downlink transmission }\end{array}$ & $\begin{array}{c}\text { Achieved full duplex transmission } \\
\text { at the AP }\end{array}$ \\
\hline$[102]$ & Enable multiple access in AmBC & $\begin{array}{c}\text { Develop an algorithm for multiplicative } \\
\text { RF transmission over channels }\end{array}$ & $\begin{array}{c}\text { Achieved higher throughput over } \\
\text { conventional AmBC }\end{array}$ \\
\hline$[103]$ & EE NOMA BackCom & $\begin{array}{c}\text { Develop a hybrid (TDMA and PD-NOMA) } \\
\text { resource allocation technique }\end{array}$ & $\begin{array}{c}\text { Achieved uplink multiple access with } \\
\text { better throughput over TDMA BackCom }\end{array}$ \\
\hline$[104]$ & $\begin{array}{c}\text { Enable uplink multiple acces } \\
\text { in BackCom }\end{array}$ & $\begin{array}{c}\text { Design a protocol based on slotted } \\
\text { ALOHA for tag operation }\end{array}$ & $\begin{array}{c}\text { Achieved multiple access with } \\
\text { less collision and higher throughput }\end{array}$ \\
\hline$[105]$ & EE NOMA BackCom & $\begin{array}{c}\text { Design a convex optimization problem } \\
\text { constrained on EH and SINR }\end{array}$ & $\begin{array}{c}\text { Achieved a more EE operation compared } \\
\text { to OMA with TDMA }\end{array}$ \\
\hline$[106]$ & EE NOMA BackCom with \\
QoS guaranty & $\begin{array}{c}\text { Design a non-convex optimization problem } \\
\text { constrained on user QoS }\end{array}$ & $\begin{array}{c}\text { Achieved higher energy efficiency } \\
\text { compared to conventional NOMA }\end{array}$ \\
\hline
\end{tabular}

operation in NOMA BackCom network, they do not guarantee $\mathrm{QoS}$ in the network operation. Hence, authors in [106] developed the first technique that maximizes EE in NOMA BackCom while guaranteeing QoS. A non-convex optimization problem was developed in order to maximize energy with constraints on user QoS requirements, order of decoding data at receiver, and tag reflection coefficient. Simulated results of the developed technique showed high energy efficiency in comparison to other NOMA BackCom.

\section{E. Modulation and Coding}

Sensing information needs to be modulated onto a carrier signal using power efficient modulation schemes. The choice of modulation scheme largely depends on the type (analog or digital) of sensing data. A suitable choice can increase the overall performance of the IoT nodes. Authors in [109] leveraged modulated backscatter at sensors to form the first wireless passive sensor network (WPSN). The WPSN uses incident RF signal to modulate sensor data and harvest energy for onboard storage thereby eliminating the need for battery. Since the harvested on-board energy is small, the authors designed a passive data retrieval protocol that clusters the nodes for transmission of data to the sink at low-power budgets. Further, authors in [110] designed a tag that uses 4-pulse amplitude modulation (PAM) for modulation in AmBC underlaying FM radio signals. The designed tag converts sensed (analog) data to digital form then modulates them onto the incident FM signal for reflection to a receiver. A microcontroller was used for switching the antenna. Since the tag uses PAM, the complexity of the receiver is drastically reduced by adopting an envelope detector for decoding received signals. A prototype of the tag operating in an indoor environment consumed $27 \mu \mathrm{W}$.

In order to leverage the advantages of OFDM in BackCom, authors in [111], [112] designed tags that leverage ambient OFDM signals as incident carriers. The authors in [111] used four sensors operating at different frequencies $(2.39,2.46$, 2.57 and $2.67 \mathrm{GHz}$ ) to collectively operate like an OFDMenabled BackCom tag. The sensors modulate sensing data using 4-quadrature amplitude modulation (4-QAM) scheme. On the other hand, authors in [112] modelled the OFDM AmBC from the spread spectrum perspective. They considered the modulation of backscatter data onto ambient OFDM signals as a multiplication operation. Authors in [113] further developed techniques to allow non-coherent BackCom with OFDM carrier. This led to lower consumption of energy and lower complexity of decoding process since synchronization with transmitter and CSI estimation are not required for signal detection. Similarly, authors in [114] developed non-coherent signal detection for AmBC. To achieve that, Manchester encoding was used to encode transmitted data at the tag. Then, an optimal decision process is employed at the receiver to detect the transmitted data. BER analysis of the AmBC system showed better performance over on-off keying (OOK) modulation.

Authors in [115] designed energy efficient prefix codes for AmBC. The code assists BackCom to take advantage of energy disparity between transmitted and received bits 0 and 1 . A prefix codebook is obtained from a derived energy minimization problem and then shared with both transmitter and receiver. Data blocks to be transmitted are broken down into smaller blocks then transmitted to the receiver. The receiver then uses the shared codebook to decode the transmitted data.

Work related to underwater (acoustic) BackCom is lagging when compared to RF BackCom and simple on-off modulation schemes are adopted. In order to enable higher order modulation, authors in [116] developed a tag the can backscatter acoustic data by switching between various reflection states. This allows increased transmitted bits per symbol through QAM and hence throughput improvement.

\section{F. Technology-assisted and Intelligent Techniques}

Since the operation of BackCom device depends on an exciter for $\mathrm{EH}$, the device suffers when the signal from the exciter is absent or low. Building on pioneering work in [117] authors in [118], [119] proposed schemes that leverage unmanned aerial vehicles (UAVs) as BSs to assist the operation of BackCom systems by providing strong excitation signals to BackCom devices while the UAVs travel through some trajectories. Authors in [118] tried to formulate an EE trajectory as an optimization problem by considering the outage probability of the BackCom system, but they considered a fixed trajectory in the problem formulation, which does not capture the practical UAV trajectory. On the other hand, authors in [119] formulated an EE non-convex optimization problem then iteratively converted it to a convex optimization problem by considering the global state information of the system in order to estimate the UAV trajectory. Nevertheless, properly obtaining the global state information is non-trivial due to the dynamic nature of the UAV. Therefore, authors in [120] leveraged a deep reinforcement learning (DRL) technique in order to combine the policy making and perception strengths of RL and deep learning (DL), respectively, to estimate an EE trajectory for 
Table X: Summary of modulation and coding techniques

\begin{tabular}{|c|c|c|c|}
\hline Reference & Aim & Implementation & Result \\
\hline$[109]$ & $\begin{array}{c}\text { BackCom assisted passive wireless } \\
\text { sensor network }\end{array}$ & $\begin{array}{c}\text { Use load modulation at the sensor } \\
\text { nodes to transmit data } \\
\text { Use a microcontroller to switch } \\
\text { between various states at the tag }\end{array}$ & $\begin{array}{c}\text { Achieved high network reliability } \\
\text { and longer life time for sensors }\end{array}$ \\
\hline$[110]$ & Enable PAM in AmBC & $\begin{array}{c}\text { Received data using low } \\
\text { complexity receiver }\end{array}$ \\
\hline$[111]$ & Enable OFDM-like BackCom & $\begin{array}{c}\text { Model modulation as multiplication } \\
\text { operation }\end{array}$ & $\begin{array}{c}\text { Successfully decoded } \\
\text { transmitted data }\end{array}$ \\
\hline$[112]$ & Enable OFDM-like BackCom & $\begin{array}{c}\text { Use four circuits operating different } \\
\text { frequencies to design 4-QAM }\end{array}$ & $\begin{array}{c}\text { Successfully decoded } \\
\text { transmitted data }\end{array}$ \\
\hline$[113]$ & Enable Non-coherent AmBC & $\begin{array}{c}\text { Leverage the spectrum structure } \\
\text { of OFDM symbols to transmit data }\end{array}$ & $\begin{array}{c}\text { Less complex and low-powered } \\
\text { receiver was used for decoding signals }\end{array}$ \\
\hline$[114]$ & Enable Non-coherent AmBC & $\begin{array}{c}\text { Develop an optimal decision policy } \\
\text { for decoding Manchester encoded } \\
\text { data from tags }\end{array}$ & $\begin{array}{c}\text { Achieved higher BER over OOK } \\
\text { with a codebook }\end{array}$ \\
\hline$[115]$ & EE coding in AmBC \\
in BackCom & $\begin{array}{c}\text { Develeped } \\
\text { wigher order modulation } \\
\text { in underwater BackCom }\end{array}$ & $\begin{array}{c}\text { Design a tag that can enable QAM } \\
\text { at the underwater tag }\end{array}$ & $\begin{array}{c}\text { Improved the throughput } \\
\text { of underwater BackCom } \\
\text { successully }\end{array}$ \\
\hline$[116]$ & & &
\end{tabular}

the UAV. In the problem formulation, they also considered BackCom device scheduling, power reflection coefficients and fairness. Results of the proposed DRL technique showed near optimal performance and gains over existing techniques. Estimating the state of a micro aerial vehicle (MAV) in a trajectory while assisting a LoRA BackCom is challenging because the BackCom devices are required to communicate over longer distances. Hence authors in [121] developed a state estimation technique called Marvel that leverages RF tags attached to the landing gears of the MAV for state estimation. To enhance the accuracy of Marvel, state initialization and calibration are done online. An MAV adopting Marvel could achieve $34 \mathrm{~cm}$ accuracy (in localization) and $4.99^{\circ}$ accuracy (in pose estimation) while travelling in long range trajectories at various speeds.

In addition to leveraging UAVs to provide RF EH source, UAVs have also been leveraged to reduce the backscatter data collection overhead [122], [123] and improve BackCom throughput [124], [125]. For a scenario that adopts a single UAV, authors in [122] modelled the trajectory design of the UAV as an optimization problem with constraints on some important parameters including UAV altitude, number of sensors, and reflection coefficient. After obtaining the optimal trajectory the sensors access the uplink channel (sensor-toUAV) using PD-NOMA in order to achieve a high throughput at the UAV. Further, authors in [123] leveraged DRL to design a technique for data collection in BackCom networks. The proposed technique divides the sensors into nodes and ensures minimum operation (flight and data collection) time for the UAV. For throughput improvement, authors in [124] leveraged the UAV as a relay for forwarding backscatter data from one node to another. The operation protocol of the UAV was modelled as a non-convex optimization problem aimed at maximizing throughput. Simulations of the protocol showed significant throughput gains. Similarly, authors in [125] developed an optimal policy for backscatter relaying network (BRN) in order to assist performance of device-todevice (D2D) networks. In BRN, an active node transmits during a particular time slot while other nodes assist its transmission through backscatter. During other slots, the prior assisting nodes may suffer from a low remaining energy. Hence the authors developed an optimal policy based on DRL for determining the operating conditions for relay nodes without affecting overall throughput. The BRN showed BackCom throughput improvement. While existing technology-assisted
BackCom techniques enhance performance of BackCom-based IoT network, they do not guaranty QoS for connected users. Hence, authors in [126] leveraged a laser powered UAV relay and RF backscatter to propose an algorithm to address the problem. At the uplink of the UAV relay, the number of connected devices and transmission from each device is maximized.

A novel technology that has shown great potential as an assistive technology for BackCom-based IoT is intelligent reflective surface (IRS) [132]. IRS can reflect ambient signals to produce multiple-channel transmissions thereby enhancing the reliability and range of reflective radio communication such as BackCom. Hence, authors in [127] leveraged IRS to assist the performance of a bistatic BackCom system. Since IRS produces many reflected signals with varying phase shifts at the receiver, a non-convex optimization model was developed to jointly synchronize the beamforming of the source and phase shifts at the IRS. This resulted in reduced power required for transmission and enhanced tag communication range.

At the receiver of an AmBC system, detecting and decoding the backscattered signal is very challenging because the reflected signal experiences high path loss, and it is interfered with at the receiver by a stronger signal directly from the ambient RF source. In order to address this issue, coherent and non-coherent techniques have been developed. Authors in [128] proposed a receiver design that involves replacing simple envelope detectors with matched filters. The authors also proposed a coherent detection of BackCom signals with OFDM carrier at the receiver. Since coherent detection requires estimating the carrier signal CSI, the matched filter attached to the backscatter device is used to estimate the carrier signal CSI. This information is then used at the receiver for joint estimation and detection of the received signal. However, high computational complexity of joint estimation and decoding prompted the design and adoption of an iterative expectation maximization (EM) algorithm. Where the duration of each iteration is proportional to OFDM symbol duration. In contrast, authors in [129] leveraged the impact of clustering in BackCom to develop an unsupervised learning based approach for signal detection at the receiver of an AmBC system, which does require channel estimation. The authors proposed a technique that mines the energy levels of received signals and then uses the result of energy mining to determine the information sent (tag symbols). Further exploiting clustering at the receiver, authors in [130] developed a constellation learning (CL) based 
Table XI: Summary of technology-assisted and intelligent techniques

\begin{tabular}{|c|c|c|c|}
\hline Reference & Aim & Implementation & Result \\
\hline$[120]$ & $\begin{array}{l}\text { EE estimation of UAV } \\
\text { trajectory }\end{array}$ & $\begin{array}{l}\text { Use DRL for EE estimation of } \\
\text { trajectory }\end{array}$ & $\begin{array}{l}\text { Achieved near optimal } \\
\text { estimation performance }\end{array}$ \\
\hline [121] & $\begin{array}{l}\text { MAV state estimation in long } \\
\text { range trajectory }\end{array}$ & $\begin{array}{l}\text { Use RF tags attached to the } \\
\text { landing gears of MAV }\end{array}$ & $\begin{array}{l}\text { Achieved high accuracy }\left(34 \mathrm{~cm} \text { and } 4.99^{0}\right) \\
\text { in localization and orientation estimation }\end{array}$ \\
\hline [122] & $\begin{array}{l}\text { Single-UAV assisted BackCom } \\
\text { data collection }\end{array}$ & $\begin{array}{l}\text { Design an optimal trajectory for UAV } \\
\text { and enable uplink multiple access }\end{array}$ & $\begin{array}{l}\text { Achieved optimal trajectory and } \\
\text { high decoding of data at the UAV }\end{array}$ \\
\hline [123] & $\begin{array}{c}\text { Multiple-UAV assisted BackCom } \\
\text { data collection }\end{array}$ & $\begin{array}{l}\text { Design techniques for minimum } \\
\text { operating time of UAV using DRL }\end{array}$ & $\begin{array}{l}\text { Achieved shortest operation time when } \\
\text { maximum (four) UAVs were deployed }\end{array}$ \\
\hline [124] & $\begin{array}{l}\text { UAV assisted throughput } \\
\text { enhancement }\end{array}$ & $\begin{array}{l}\text { Design an operating protocol } \\
\text { for UAV as a relay }\end{array}$ & Achieved high throughput gain \\
\hline [125] & $\begin{array}{l}\text { D2D-relaying assisted throughput } \\
\text { improvement }\end{array}$ & $\begin{array}{l}\text { Design an optimal policy for setting } \\
\text { operating condition for relay nodes }\end{array}$ & $\begin{array}{c}\text { Improved BackCom network } \\
\text { throughput }\end{array}$ \\
\hline [126] & $\begin{array}{l}\text { QoS guaranty in UAV-assisted } \\
\text { BackCom based IoT }\end{array}$ & $\begin{array}{l}\text { Design techniques to ensure UAV relaying } \\
\text { through BackCom and multiple access } \\
\text { to guaranty QoS }\end{array}$ & $\begin{array}{l}\text { Achieved higher arrival rate from } \\
\text { IoT nodes and higher number of } \\
\text { connected IoT nodes }\end{array}$ \\
\hline [127] & IRS assisted BackCom & $\begin{array}{l}\text { Jointly optimize phase shifts at } \\
\text { the IRS and transmitter beamforming }\end{array}$ & $\begin{array}{l}\text { Reduced transmitter power at the } \\
\text { source and enhanced the tag's range }\end{array}$ \\
\hline [128] & $\begin{array}{l}\text { Intelligent coherent signal } \\
\text { detection at receiver }\end{array}$ & $\begin{array}{l}\text { Develop unsupervised learning algorithm } \\
\text { for joint signal estimation and decoding }\end{array}$ & $\begin{array}{l}\text { Achieved coherent detection } \\
\text { with a low-cost receiver }\end{array}$ \\
\hline [129] & $\begin{array}{l}\text { Intelligent signal detection } \\
\text { at receiver }\end{array}$ & $\begin{array}{l}\text { Exploit the clustering phenomenon } \\
\text { shown by received signals }\end{array}$ & $\begin{array}{l}\text { Achieved non-coherent signal } \\
\text { detection }\end{array}$ \\
\hline [130] & $\begin{array}{l}\text { Intelligent signal detection } \\
\text { at receiver }\end{array}$ & $\begin{array}{l}\text { Assign labels to transmissions from } \\
\text { the tag and unsupervised learning to } \\
\text { decode data at receiver }\end{array}$ & $\begin{array}{c}\text { Achieved non-coherent signal } \\
\text { detection }\end{array}$ \\
\hline [131] & $\begin{array}{l}\text { Avoid interference at the } \\
\text { receiver }\end{array}$ & $\begin{array}{l}\text { Model the operation of devices and } \\
\text { use } R L \text { to learn optimal policy }\end{array}$ & $\begin{array}{l}\text { Increased receiver performance in } \\
\text { the presence of interferer }\end{array}$ \\
\hline
\end{tabular}

technique that exploits the channel diversity of a multiple antenna receiver to detect tag symbols. Rather than mining energy of the received signals, the authors assigned labels to each packet at the tag. So, the received signal forms a constellation based on the labels attached at the tag. An EM algorithm that is constrained by the modulation technique at the receiver is then used to detect the signal received. The CL based technique showed good results that are comparable to that of an optimal detector with perfect CSI.

Rather than developing green techniques for signal detection in the presence of interference, authors in [131] developed a RL based technique for interference avoidance. The authors modelled the interaction between a BackCom system and an interferer as a game. Then, they considered EH and backscattering time to develop the utility functions. For each sub-game, Q-learning is used to obtain the optimal policy for the game. Results of the proposed technique showed better performance compared to other interference avoidance models that assume random and fixed backscattering times.

\section{G. Device Cooperation}

Conventional AmBC systems have employed various techniques at the receiver in order to decode information contained in the backscattered signal while suppressing the interference from the ambient source signal. Though such techniques have shown promising results, they may not be spectrum or energy efficient. Hence authors in [133] designed a cooperative AmBC model that is able to simultaneously decode modulated information received from the backscatter device and that from the source. They employed spread spectrum and spectrum sharing techniques to develop models of the cooperative receiver for both flat and frequency selective fading channels. Also, maximum likelihood detectors were used to detect the signals considering the fact that a backscattered signal contains the source signal. Extensive simulations showed good detection of the two signals at the receiver.

In order to address the problem of limited energy in IoT nodes, authors in [134] developed a novel EE cooperative communication scheme (EECCS). The scheme combines energy beamforming and $\mathrm{AmBC}$ to ensure availability of sufficient energy for the sensor nodes, thereby increasing the amount of sensing data at the sink (receiver). The scheme was modelled as a resource allocation problem in which the optimal resource allocation policy for various parameters (node transmission power and sensing data size of nodes) is determined. The scheme showed improved energy efficiency over existing sensor networks and increased amount of sensing data at the receiver. Despite developing techniques for ensuring sufficient energy at $\mathrm{AmBC}$ nodes, some of the nodes may still experience challenges in signal detection due to low energy. This could be as a result of a long distance between the node and the source or sink. To address this problem, authors in [135] developed a technique called backscatter cooperation-NOMA (BC-NOMA) that leverages the excess downlink (source-to-tag) RF power at one node to assist signal detection in another node with low available power. Comparison of BC-NOMA with other NOMA cooperation schemes showed a higher transmission reliability. To further assist AmBC nodes with low power, authors in [136] proposed a novel technique that collectively assists nodes with low power. In this technique, the nodes are first grouped into near and far regions based on their distance to the sink. Then nodes in the near region are associated with nodes in the far region based on their individual energy levels to assist one-another. The proposed technique showed more successful grouping of nodes and higher number of successful transmissions when compared in cooperative NOMA with random node pairing.

\section{H. Node Architecture Re-design}

Since its inception, passive RFIDs have been deployed in various low power applications. In such applications, the tag operation is sustained by harvesting RF energy from the signal coming from the reader. In order to enhance RF energy harvesting, authors in [137] designed an analog frontend for passive RFID. The design consists of a power rectifier for converting RF energy to a direct current (DC) voltage, an amplitude shift keying (ASK) demodulator for decoding the reader signal, a backscatter modulator for transmitting information 
Table XII: Summary of device cooperation techniques

\begin{tabular}{|c|c|c|c|}
\hline Reference & Aim & Implementation & Result \\
\hline$[133]$ & $\begin{array}{c}\text { Interference-free backscatter } \\
\text { signal detection }\end{array}$ & $\begin{array}{c}\text { Develop a technique to simultaneously decode } \\
\text { backscatter and RF source signal }\end{array}$ & $\begin{array}{c}\text { Successful detection of } \\
\text { both signals }\end{array}$ \\
\hline$[134]$ & $\begin{array}{c}\text { Increase available power of } \\
\text { nodes in AmBC }\end{array}$ & $\begin{array}{c}\text { Design a scheme that uses cooperative } \\
\text { energy beamforming for EH }\end{array}$ & $\begin{array}{c}\text { Improd energy efficiency } \\
\text { in AmBC }\end{array}$ \\
\hline$[135]$ & $\begin{array}{c}\text { Assist signal detection in } \\
\text { low-power nodes }\end{array}$ & $\begin{array}{c}\text { Design a technique for transferring } \\
\text { excess available power between nodes }\end{array}$ & $\begin{array}{c}\text { Achieved higher transmission reliability } \\
\text { in low power nodes }\end{array}$ \\
\hline$[136]$ & $\begin{array}{c}\text { Collectively assist low power } \\
\text { nodes with similar energy }\end{array}$ & $\begin{array}{c}\text { Design techniques that will group and } \\
\text { cooperate nodes based on their energy } \\
\text { levels and distance to sink }\end{array}$ & $\begin{array}{c}\text { Achieved more successful transmissions } \\
\text { compared to random cooperative NOMA }\end{array}$ \\
\hline
\end{tabular}

Table XIII: Summary of architecture re-design techniques

\begin{tabular}{|c|c|c|c|}
\hline Reference & Aim & Implementation & Result \\
\hline [137] & $\begin{array}{l}\text { Improve EH at the tag } \\
\text { of passive RFID }\end{array}$ & $\begin{array}{l}\text { Re-design the analog RF frontend } \\
\text { of RFID tag }\end{array}$ & $\begin{array}{l}\text { Achieved a high }(20 \%) \mathrm{RF} \\
\text { conversion rate }\end{array}$ \\
\hline [138] & $\begin{array}{l}\text { Improve EH with a } \\
\text { compact-sized tag }\end{array}$ & $\begin{array}{l}\text { Design a switching mechanism for } \\
\text { use with a single-antenna tag }\end{array}$ & $\begin{array}{l}\text { Achieved a high }(20 \%) \mathrm{RF} \\
\text { conversion rate }\end{array}$ \\
\hline [139] & $\begin{array}{l}\text { Enable spatial modulation } \\
\text { in AmBC }\end{array}$ & $\begin{array}{l}\text { A patch antenna with its switching } \\
\text { circuit was used as RF frontend }\end{array}$ & $\begin{array}{l}\text { Achieved spatial modulation by } \\
\text { switching between four modes }\end{array}$ \\
\hline [140] & Enable mm-wave BackCom & $\begin{array}{l}\text { Design the mm-wave RF frontend } \\
\text { with simple components }\end{array}$ & $\begin{array}{l}\text { Consumed low }(72 \mu \mathrm{W}) \text { power and } \\
\text { achieved high data rate }(1.2 \mathrm{Gbps})\end{array}$ \\
\hline [141] & $\begin{array}{l}\text { Digital synthesis of BackCom } \\
\text { in RFID }\end{array}$ & $\begin{array}{l}\text { Design a digital baseband controller } \\
\text { compatible with RFID }\end{array}$ & $\begin{array}{l}\text { Transmitted packets to RFID } \\
\text { reader at low }(5.76 \mu \mathrm{W}) \text { power }\end{array}$ \\
\hline [142] & $\begin{array}{l}\text { Digital synthesis of OFDM } \\
\text { BackCom }\end{array}$ & $\begin{array}{l}\text { Design a digital baseband using RF } \\
\text { switches for OFDM signal synthesis }\end{array}$ & $\begin{array}{l}\text { Achieved higher number of OFDM } \\
\text { carriers over analog-based } \\
\text { signal synthesis }\end{array}$ \\
\hline [143] & $\begin{array}{c}\text { End-to-end EE BackCom } \\
\text { transmitter }\end{array}$ & $\begin{array}{l}\text { Re-design the BackCom tag by } \\
\text { eliminating power-hungry components }\end{array}$ & $\begin{array}{l}\text { Re-designed tag consumed } \\
\text { low }(37 \mu \mathrm{W}) \text { power }\end{array}$ \\
\hline [144] & EE BackCom receiver & $\begin{array}{l}\text { Design simple signal detectors at } \\
\text { the receiver with analog components }\end{array}$ & $\begin{array}{l}\text { Could decode backscatter signal } \\
\text { without interference }\end{array}$ \\
\hline [145] & Re-design software of BackCom & $\begin{array}{l}\text { Design an energy-aware } \\
\text { scalable network }\end{array}$ & $\begin{array}{l}\text { Could vary the size of BackCom packets } \\
\text { down to bit level based on energy }\end{array}$ \\
\hline
\end{tabular}

from the tag, and a voltage limiter to protect the tag from high incident power. The designed frontend achieved $20 \%$ power conversion while only consuming $1 \mu \mathrm{W}$ at $1 \mathrm{~V}$. Similarly, authors in [138] re-designed the RF frontend of BackCom nodes by incorporating a switching mechanism. This enables the nodes to perform energy harvesting and backscatter with a single antenna, thereby eliminating power overhead caused by using two antennas for the tag's operation. Further, authors in [139] re-designed the RF frontend of a tag by adopting a reconfigurable, compact and energy efficient patch antenna suitable for spatial modulation in AmBC applications at 3.5 GHz. An extensive characteristic mode analysis (CMA) and feeding circuit design were adopted to ensure optimal design of the antenna. Motivated by the need to provide BackCom with higher data rates and smaller circuit size, authors in [140] developed a mm-wave RF backscatter frontend. The circuit was designed with a transistor and a low order modulator for operation within the $20-26 \mathrm{GHz}$ band. At peak dynamic operating conditions, the circuit consumed a low $(72 \mu \mathrm{W})$ power and achieved a high (1.2 Gbps) data rate.

While the previous works re-designed the analog frontend, authors in [141] designed an EE digital baseband controller (DBC) that is compatible with the widely used EPC Class-1 Gen-2 protocol of RFID. In the DBC design, asynchronous counting, clock gating and disabled simultaneous decoding and backscatter operation at the analog frontend were adopted to reduce energy consumption. The designed DBC consumed $5.76 \mu \mathrm{W}$ at $1.2 \mathrm{~V}$. Similarly, authors in [142] designed an efficient hardware architecture for complete digital synthesis of OFDM backscatter. The designed hardware adopts digital RF switching to enable OFDM rather than the power-hungry ADCs (analog-to-digital converter) and DAC (digital-to-analog converters). The designed tag was able to synthesize more OFDM carriers at the tag thereby improving the performance of BackCom receiver in challenging multipath environments.

In order to achieve end-to-end EE operation, authors in
[143] completely re-designed the BackCom node architecture in order to reduce the computational overhead it incurs. In the end-to-end re-design, energy hungry computational blocks between the sensor and RF frontend were analysed then eliminated to form a minimal low energy consuming node. In order to ensure scalable operation of the node, a medium access control (MAC) layer that allows flexible data rate at the reader was designed. The architecture performed better than existing architecture and consumed $37 \mu \mathrm{W}$. Since the receiver on $\mathrm{AmBC}$ experiences challenges in decoding backscatter signals due to interference and low signal to noise ratio (SNR), authors in [144] leveraged analog components to design an energy efficient signal detection architecture for AmBC systems. The architecture is inspired by stochastic resonance (SR), where nonlinear filtering techniques are used to suppress the noise signal and enhance the backscatter signal. Then, simple averaging circuits and automatic threshold detection are used to detect the backscatter signal.

Re-designing the software architecture of BackCom node, authors in [145] proposed an energy-aware scalable network stack (QuarkNet) that optimizes the operation of BackCom nodes by scaling (down to bit level) the size of packet to be transmitted by the BackCom node. The extent of scaling depends on the available energy at the BackCom node rather than available sensing data packets.

\section{Challenges and Possible Solutions}

In this section, we discuss some of the challenges and possible solutions to the adoption of BackCom for joint communication and sensing in IoT applications.

\section{A. Security}

Applications employing BackCom for joint sensing and communication may sometimes transmit sensitive personalized information (such as a person's health status or discussions in 
a meeting), which could lead to a breach of privacy if intercepted. However the low power budgets of BackCom devices pose a bottleneck to securing BackCom because power hungry security techniques cannot work with such green budgets. As discussed, techniques have been developed for securing BackCom devices against eavesdropping and jamming attacks but BackCom signals are still susceptible to leakages [78]. A possible solution to this problem is securing the reflected signal at the tag using unique signatures generated based on a unique features of the tag's operation (context). Another solution could involve analyzing the physical layer properties of the BackCom system. For instance, authors in [146] jointly considered the outage probability (OP) and interference probability (IP) of cognitive AmBC (C-AmBC) to develop energy efficient security techniques to prevent eavesdropping of broadcast BackCom signals.

\section{B. Interference}

Another challenge to deployment of BackCom-based IoT is the interference at the receiver from the source signal. This causes errors when decoding the backscattered signal since the source signal and the reflected signal are on the same channel. Though techniques for interference mitigation and avoidance were developed, another solution to this problem is deploying BackCom tags that are able to shift the reflected signal to another channel that can also be detected and decoded by the receiver. Authors in [46], [147] have developed techniques that can shift a backscatter signal to another channel. Similarly, authors in [148] developed backscatter tags that can use a single incident carrier with two transistors biased differently to generate multiple carrier signals. Another solution is utilizing the periodicity of carrier signals to suppress interference. In that direction, authors in [149] leveraged the periodic nature of sinusoidal carrier signals to suppress interference in bistatic BackCom by designing an optimal detector at the receiver.

\section{Instability of ambient signals}

Though ambient signals have been leveraged for BackCom with promising results, the ambient sources from which the tags are excited are not always stable. For instance, network downtimes are experienced in FM radio and cellular BSs. During those downtimes, there is very low or a total absence of excitation signal. Hence, operation of AmBC-based IoT devices underlaying those infrastructures could be severely degraded. Possible solutions to this challenge include employing intelligent techniques that can enhance utilization of such ambient signals [150], harvesting energy from other sources

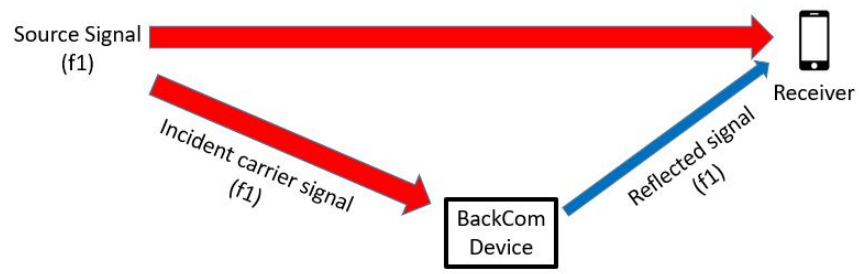

Figure 5: Interference in BackCom-based IoT at the tag [151], and generating low power carrier signals at the tag to modulate and backscatter sensing data [152], [153].

\section{Future Application Scenarios}

This section explains possible future applications of BackCom-based green IoT due to its suitability as an enabling technology in such applications.

\section{A. Healthcare}

Rendering healthcare to elderly and highly disabled persons requires deploying IoT devices (wearable and non-wearable) that can be used for very long periods of time without need for charging or replacement of batteries. Hence developing very small, lightweight and battery-free health tracking devices open a wide range of future applications. For patients that are bedridden, BackCom-based IoT could be used to measure posture, breathing, temperature, etc. This application scenario is shown in Figure 6. Furthermore, the battery-free potential of BackCom based green IoT can be leveraged to develop small and minimally invasive medical implants for use during long duration operations or for endoscopic treatments.

\section{B. Transportation}

The next generation of transportation media will incorporate intelligent sensors. These intelligent sensors will interact with road signs, building surfaces and nearby vehicles to ensure safe traveling. It also promises to provide passengers with invehicle entertainment and comfort. As vehicles increasingly adopt renewable energy (batteries) as replacement to fossil fuels, development of green BackCom based IoT sensors for monitoring the interior and exterior environments of the vehicle will become more important. Also, with BackCombased IoT the tasks of processing the large amount of data generated from intelligent transportation could be delegated to more powerful and efficient computational devices at the network edges and in the cloud, further enabling green intelligent transportation. Hence, applications that enable intelligent transportation without high computational overhead will be made possible in the future through adoption of BackCombased IoT.

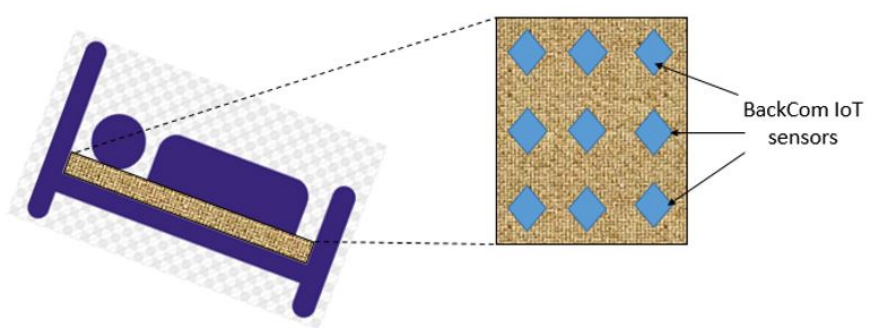

Figure 6: Monitoring bedridden patients with BackCom-based IoT 


\section{Machine Type Communication (MTC)}

Next generation of machines will incorporate BackCombased green IoT sensors that will enable one machine to sense its operating and environmental conditions and transmit such information to another machine to facilitate intelligent decision-making based on such information. These applications will allow collective and individual machines to perform joint sensing and communication operation to enhance the performance of large plants. Discussions on potential of BackCom in MTC for current and future generations of wireless networks are presented by authors in [154] [155]. Further, the first theoretical model and a feasibility study of deploying large scale BackCom tags for massive IoT operation in an urban area are presented by authors in [156].

\section{Smart city applications}

Smart city applications promise to enable communication between infrastructures such as buildings and bill boards, and humans through sensors. This could be leveraged to enhance the safety of humans living in cities. For instance, IRS can be attached on the walls of tall buildings to monitor the structural health of building. When the building is vibrating abnormally, the reflected signals from the building could be used to transmit such information in a timely manner. The same scenario could be applied to monitoring structural health of a bridge.

\section{CONCLUSION}

BackCom is a promising technology for enabling joint lowpower sensing and communication applications in the current and next generation of IoT. In this article, we have presented a comprehensive study of BackCom as a single-infrastructure for enabling joint green sensing and communications. We have described the operation of BackCom, its various categories and their applications in IoT. We have further provided details on the evolution of BackCom, merits of BackCom-based IoT and some state-of-the-art applications of BackCom in IoT. We have given a detailed survey of existing techniques that were designed to ensure that BackCom-based IoT operates at green budgets. Further, some existing challenges of BackCom-based IoT have been presented. Finally, we have suggested some possible future applications of BackCom-based IoT.

\section{ACKNOWLEDGMENT}

This research was supported in part by the China NSFC Grant (U2001207, 61872248), Guangdong NSF 2017A030312008, Shenzhen Science and Technology Foundation (No. ZDSYS20190902092853047), the Project of DEGP (No.2019KCXTD005, R2020A045), the Guangdong "Pearl River Talent Recruitment Program" under Grant 2019ZT08X603. Kaishun $\mathrm{Wu}$ is the corresponding author.

\section{REFERENCES}

[1] C. S. Abella, S. Bonina, A. Cucuccio, S. D'Angelo, G. Giustolisi, A. D. Grasso, A. Imbruglia, G. S. Mauro, G. A. Nastasi, G. Palumbo et al., "Autonomous energy-efficient wireless sensor network platform for home/office automation," IEEE Sensors Journal, vol. 19, no. 9, pp. 3501-3512, 2019.
[2] R. Morello, C. De Capua, G. Fulco, and S. C. Mukhopadhyay, "A smart power meter to monitor energy flow in smart grids: The role of advanced sensing and iot in the electric grid of the future," IEEE Sensors Journal, vol. 17, no. 23, pp. 7828-7837, 2017.

[3] F. Zhu, Y. Lv, Y. Chen, X. Wang, G. Xiong, and F.-Y. Wang, "Parallel transportation systems: toward iot-enabled smart urban traffic control and management," IEEE Transactions on Intelligent Transportation Systems, vol. 21, no. 10, pp. 4063-4071, 2019.

[4] H. Li, K. Ota, and M. Dong, "Always connected things: Building disaster resilience iot communications," in 2019 IEEE 25th International Conference on Parallel and Distributed Systems (ICPADS). IEEE, 2019, pp. 570-577.

[5] "Global iot market will grow to 24.1 billion devices in 2030 , generating \$ 1.5 trillion annual revenue," https://www.prnewswire.com/ news-releases/global-iot-market-will-grow-to-24-1.html, accessed: 2021-02-18.

[6] Y. Li, S. Chen, W. Ye, and F. Lin, "A joint low-power cell search and frequency tracking scheme in nb-iot systems for green internet of things," Sensors, vol. 18, no. 10, p. 3274, 2018.

[7] A. S. H. Abdul-Qawy, N. M. S. Almurisi, and S. Tadisetty, "Classification of energy saving techniques for iot-based heterogeneous wireless nodes," Procedia Computer Science, vol. 171, pp. 2590-2599, 2020.

[8] A. Izaddoost and M. Siewierski, "Energy efficient data transmission in iot platforms," Procedia Computer Science, vol. 175, pp. 387-394, 2020.

[9] X. Liu and N. Ansari, "Toward green iot: Energy solutions and key challenges," IEEE Communications Magazine, vol. 57, no. 3, pp. 104110, 2019.

[10] D. Wang, W. Tang, X. Yang, and W. Feng, "Cryptographic algorithm invocation in ipsec: Guaranteeing the communication security in the southbound interface of sdn networks," in International Conference on Communications and Networking in China. Springer, 2018, pp. 583592.

[11] O. L. López, H. Alves, R. D. Souza, S. Montejo-Sánchez, E. M. Fernandez, and M. Latva-aho, "Massive wireless energy transfer: Enabling sustainable iot towards $6 \mathrm{~g}$ era," IEEE Internet of Things Journal, 2021.

[12] P. X. Nguyen, D.-H. Tran, O. Onireti, P. T. Tin, S. Q. Nguyen, S. Chatzinotas, and H. V. Poor, "Backscatter-assisted data offloading in ofdma-based wireless powered mobile edge computing for iot networks," IEEE Internet of Things Journal, 2021.

[13] U. Ha, J. Leng, A. Khaddaj, and F. Adib, "Food and liquid sensing in practical environments using rfids," in 17th $\{$ USENIX\} Symposium on Networked Systems Design and Implementation (\{NSDI\} 20), 2020, pp. 1083-1100.

[14] V. Talla, B. Kellogg, S. Gollakota, and J. R. Smith, "Battery-free cellphone," Proceedings of the ACM on Interactive, Mobile, Wearable and Ubiquitous Technologies, vol. 1, no. 2, pp. 1-20, 2017.

[15] P. Wang, L. Feng, G. Chen, C. Xu, Y. Wu, K. Xu, G. Shen, K. Du, G. Huang, and X. Liu, "Renovating road signs for infrastructure-tovehicle networking: A visible light backscatter communication and networking approach," in Proceedings of the 26th Annual International Conference on Mobile Computing and Networking, 2020, pp. 1-13.

[16] R. Ghaffarivardavagh, S. S. Afzal, O. Rodriguez, and F. Adib, "Ultrawideband underwater backscatter via piezoelectric metamaterials," in Proceedings of the Annual conference of the ACM Special Interest Group on Data Communication on the applications, technologies, architectures, and protocols for computer communication, 2020, pp. 722-734.

[17] A. Varshney, A. Soleiman, L. Mottola, and T. Voigt, "Battery-free visible light sensing," in Proceedings of the 4th ACM Workshop on Visible Light Communication Systems, 2017, pp. 3-8.

[18] B. Paul, A. R. Chiriyath, and D. W. Bliss, "Survey of rf communications and sensing convergence research," IEEE Access, vol. 5, pp. 252-270, 2016.

[19] W. Zhang, Y. Qin, W. Zhao, M. Jia, Q. Liu, R. He, and B. Ai, "A green paradigm for internet of things: Ambient backscatter communications," China Communications, vol. 16, no. 7, pp. 109-119, 2019.

[20] D. Ma, G. Lan, M. Hassan, W. Hu, and S. K. Das, "Sensing, computing, and communications for energy harvesting iots: A survey," IEEE Communications Surveys \& Tutorials, vol. 22, no. 2, pp. 1222-1250, 2019.

[21] V. Gupta, S. Tripathi, and S. De, "Green sensing and communication: A step towards sustainable iot systems," Journal of the Indian Institute of Science, vol. 100, no. 2, pp. 383-398, 2020. 
[22] W. B. Qaim, A. Ometov, A. Molinaro, I. Lener, C. Campolo, E. S. Lohan, and J. Nurmi, "Towards energy efficiency in the internet of wearable things: A systematic review," IEEE Access, 2020.

[23] G. D. Durgin, "Balanced codes for more throughput in rfid and backscatter links," in 2015 IEEE International Conference on RFID Technology and Applications (RFID-TA). IEEE, 2015, pp. 65-70.

[24] G. Wang, C. Qian, K. Cui, H. Ding, H. Cai, W. Xi, J. Han, and J. Zhao, "A (near) zero-cost and universal method to combat multipaths for rfid sensing," in 2019 IEEE 27th International Conference on Network Protocols (ICNP). IEEE, 2019, pp. 1-4.

[25] M. B. Akbar, C. Qi, M. Alhassoun, and G. D. Durgin, "Orientation sensing using backscattered phase from multi-antenna tag at $5.8 \mathrm{ghz}$," in 2016 IEEE International Conference on RFID (RFID). IEEE, 2016, pp. $1-8$.

[26] J. Kimionis, A. Bletsas, and J. N. Sahalos, "Bistatic backscatter radio for tag read-range extension," in 2012 IEEE International Conference on RFID-Technologies and Applications (RFID-TA). IEEE, 2012, pp. 356-361.

[27] _ "Bistatic backscatter radio for power-limited sensor networks," in 2013 IEEE Global Communications Conference (GLOBECOM). IEEE, 2013, pp. 353-358.

[28] S.-N. Daskalakis, J. Kimionis, A. Collado, M. M. Tentzeris, and A. Georgiadis, "Ambient fm backscattering for smart agricultural monitoring," in 2017 IEEE MTT-S International Microwave Symposium (IMS). IEEE, 2017, pp. 1339-1341.

[29] V. Liu, A. Parks, V. Talla, S. Gollakota, D. Wetherall, and J. R. Smith, "Ambient backscatter: Wireless communication out of thin air," $A C M$ SIGCOMM Computer Communication Review, vol. 43, no. 4, pp. 3950,2013

[30] M. U. Sheikh, F. Jameel, H. Yiğitler, X. Wang, and R. Jäntti, "Monostatic backscatter communication in urban microcellular environment using cellular networks," in 2020 IEEE Wireless Communications and Networking Conference (WCNC). IEEE, 2020, pp. 1-6.

[31] C. He, S. Chen, H. Luan, X. Chen, and Z. J. Wang, "Monostatic mimo backscatter communications," IEEE Journal on Selected Areas in Communications, vol. 38, no. 8, pp. 1896-1909, 2020

[32] J. Guo, S. Durrani, and X. Zhou, "Monostatic backscatter system with multi-tag to reader communication," IEEE Transactions on Vehicular Technology, vol. 68, no. 10, pp. 10320-10324, 2019.

[33] M. Katanbaf, A. Saffari, and J. R. Smith, "Receiver selectivity limits on bistatic backscatter range," in 2020 IEEE International Conference on RFID (RFID). IEEE, 2020, pp. 1-8.

[34] M. Hua, L. Yang, C. Li, Z. Zhu, and I. Lee, "Bistatic backscatter communication: Shunt network design," IEEE Internet of Things Journal, 2020.

[35] S. Zhang, W. Wang, S. Tang, S. Jin, and T. Jiang, "Robot-assisted backscatter localization for iot applications," IEEE Transactions on Wireless Communications, vol. 19, no. 9, pp. 5807-5818, 2020.

[36] X. Cao, Z. Song, B. Yang, M. A. ElMossallamy, L. Qian, and Z. Han, "A distributed ambient backscatter mac protocol for internet-of-things networks," IEEE Internet of Things Journal, vol. 7, no. 2, pp. 14881501, 2019.

[37] D. M. Mughal, T. Mahboob, and M. Y. Chung, "Capacity analysis of wirelessly powered backscatter communications for green iot," in 2019 25th Asia-Pacific Conference on Communications (APCC). IEEE, 2019, pp. 485-489.

[38] G. Brooker and J. Gomez, "Lev termen's great seal bug analyzed," IEEE Aerospace and Electronic Systems Magazine, vol. 28, no. 11, pp. 4-11, 2013.

[39] H. Stockman, "Communication by means of reflected power," Proceedings of the IRE, vol. 36, no. 10, pp. 1196-1204, 1948.

[40] V. Chawla and D. S. Ha, "An overview of passive rfid" IEEE Communications Magazine, vol. 45, no. 9, pp. 11-17, 2007.

[41] J. Li, A. Liu, G. Shen, L. Li, C. Sun, and F. Zhao, "Retro-vlc: Enabling battery-free duplex visible light communication for mobile and iot applications," in Proceedings of the 16th International Workshop on Mobile Computing Systems and Applications, 2015, pp. 21-26.

[42] V. Iyer, J. Chan, and S. Gollakota, "3d printing wireless connected objects," ACM Transactions on Graphics (TOG), vol. 36, no. 6, pp. $1-13,2017$.

[43] Z. Luo, W. Wang, J. Xiao, Q. Huang, T. Jiang, and Q. Zhang, "Authenticating on-body backscatter by exploiting propagation signatures," Proceedings of the ACM on Interactive, Mobile, Wearable and Ubiquitous Technologies, vol. 2, no. 3, pp. 1-22, 2018.

[44] J. Jang and F. Adib, "Underwater backscatter networking," in Proceedings of the ACM Special Interest Group on Data Communication, 2019, pp. $187-199$
[45] A. Galisteo, A. Varshney, and D. Giustiniano, "Two to tango: Hybrid light and backscatter networks for next billion devices," in Proceedings of the 18th International Conference on Mobile Systems, Applications, and Services, 2020, pp. 80-93.

[46] P. Zhang, M. Rostami, P. Hu, and D. Ganesan, "Enabling practical backscatter communication for on-body sensors," in Proceedings of the 2016 ACM SIGCOMM Conference, 2016, pp. 370-383.

[47] V. Ranganathan, S. Gupta, J. Lester, J. R. Smith, and D. Tan, "Rf bandaid: A fully-analog and passive wireless interface for wearable sensors," Proceedings of the ACM on Interactive, Mobile, Wearable and Ubiquitous Technologies, vol. 2, no. 2, pp. 1-21, 2018.

[48] D. Vasisht, G. Zhang, O. Abari, H.-M. Lu, J. Flanz, and D. Katabi, "In-body backscatter communication and localization," in Proceedings of the 2018 Conference of the ACM Special Interest Group on Data Communication, 2018, pp. 132-146.

[49] X. Fan, L. Shangguan, R. Howard, Y. Zhang, Y. Peng, J. Xiong, Y. Ma, and X.-Y. Li, "Towards flexible wireless charging for medical implants using distributed antenna system," in Proceedings of the 26th Annual International Conference on Mobile Computing and Networking, 2020, pp. 1-15.

[50] B. Kellogg, V. Talla, and S. Gollakota, "Bringing gesture recognition to all devices," in 11th \{USENIX\} Symposium on Networked Systems Design and Implementation (\{NSDI\} 14), 2014, pp. 303-316.

[51] Z. Chi, Y. Yao, T. Xie, X. Liu, Z. Huang, W. Wang, and T. Zhu, "Ear: Exploiting uncontrollable ambient rf signals in heterogeneous networks for gesture recognition," in Proceedings of the 16th ACM conference on embedded networked sensor systems, 2018, pp. 237-249.

[52] J. Ryoo, Y. Karimi, A. Athalye, M. Stanaćević, S. R. Das, and P. Djurić, "Barnet: Towards activity recognition using passive backscattering tag-to-tag network," in Proceedings of the 16th Annual International Conference on Mobile Systems, Applications, and Services, 2018, pp. 414-427.

[53] K. Joshi, D. Bharadia, M. Kotaru, and S. Katti, "Wideo: Finegrained device-free motion tracing using $\{\mathrm{RF}\}$ backscatter," in 12th $\{$ USENIX\} Symposium on Networked Systems Design and Implementation ( $\{$ NSDI $\}$ 15), 2015, pp. 189-204.

[54] X. Meng, L. Feng, X. Yin, H. Zhou, C. Sheng, C. Wang, A. Du, and $\mathrm{L}$. Xu, "Sentence-level sign language recognition using rf signals," in 2019 6th International Conference on Behavioral, Economic and SocioCultural Computing (BESC). IEEE, 2019, pp. 1-6.

[55] J. Wang, C. Pan, H. Jin, V. Singh, Y. Jain, J. I. Hong, C. Majidi, and S. Kumar, "Rfid tattoo: A wireless platform for speech recognition," Proceedings of the ACM on Interactive, Mobile, Wearable and Ubiquitous Technologies, vol. 3, no. 4, pp. 1-24, 2019.

[56] C. Konstantopoulos, E. Kampianakis, E. Koutroulis, and A. Bletsas, "Wireless sensor node for backscattering electrical signals generated by plants," in SENSORS, 2013 IEEE. IEEE, 2013, pp. 1-4.

[57] S. N. Daskalakis, G. Goussetis, S. D. Assimonis, M. M. Tentzeris, and A. Georgiadis, "A uw backscatter-morse-leaf sensor for low-power agricultural wireless sensor networks," IEEE Sensors Journal, vol. 18 , no. 19 , pp. 7889-7898, 2018.

[58] C. Konstantopoulos, E. Koutroulis, N. Mitianoudis, and A. Bletsas, "Converting a plant to a battery and wireless sensor with scatter radio and ultra-low cost," IEEE Transactions on Instrumentation and Measurement, vol. 65, no. 2, pp. 388-398, 2015.

[59] G. Vougioukas and A. Bletsas, " $24 \mu$ watt $26 \mathrm{~m}$ range batteryless backscatter sensors with fm remodulation and selection diversity," in 2017 IEEE International Conference on RFID Technology \& Application (RFID-TA). IEEE, 2017, pp. 237-242.

[60] S.-N. Daskalakis, S. D. Assimonis, E. Kampianakis, and A. Bletsas, "Soil moisture scatter radio networking with low power," IEEE Transactions on Microwave Theory and Techniques, vol. 64, no. 7, pp. 23382346, 2016.

[61] J. Wang, L. Chang, S. Aggarwal, O. Abari, and S. Keshav, "Soil moisture sensing with commodity rfid systems," in Proceedings of the 18th International Conference on Mobile Systems, Applications, and Services, 2020, pp. 273-285.

[62] W. U. Khan, F. Jameel, N. Kumar, R. Jäntti, and M. Guizani, "Backscatter-enabled efficient v2x communication with non-orthogonal multiple access," IEEE Transactions on Vehicular Technology, vol. 70, no. 2, pp. 1724-1735, 2021.

[63] B. Ji, Z. Chen, S. Mumtaz, J. Liu, Y. Zhang, J. Zhu, and C. Li, "Swipt enabled intelligent transportation systems with advanced sensing fusion," IEEE Sensors Journal, 2020.

[64] C. Gao, Y. Li, and X. Zhang, "Livetag: Sensing human-object interaction through passive chipless wifi tags," in 15th \{USENIX\} Symposium 
on Networked Systems Design and Implementation (\{NSDI\} 18), 2018, pp. 533-546.

[65] A. Wang, V. Iyer, V. Talla, J. R. Smith, and S. Gollakota, " $\{F M\}$ backscatter: Enabling connected cities and smart fabrics," in 14th \{USENIX\} Symposium on Networked Systems Design and Implementation ( $\{$ NSDI $\} 17), 2017$, pp. 243-258.

[66] S. Chen, M. Zhang, J. Zhao, W. Gong, and J. Liu, "Reliable and practical bluetooth backscatter with commodity devices," IEEE/ACM Transactions on Networking, 2021.

[67] X. Lu, H. Jiang, D. Niyato, D. I. Kim, and P. Wang, "Analysis of wireless-powered device-to-device communications with ambient backscattering," in 2017 IEEE 86th Vehicular Technology Conference (VTC-Fall). IEEE, 2017, pp. 1-6.

[68] L. Xu, K. Zhu, R. Wang, and S. Gong, "Performance analysis of ambient backscatter communications in rf-powered cognitive radio networks," in 2018 IEEE Wireless Communications and Networking Conference (WCNC). IEEE, 2018, pp. 1-6.

[69] Z. B. Zawawi, Y. Huang, and B. Clerckx, "Multiuser wirelessly powered backscatter communications: Nonlinearity, waveform design, and sinr-energy tradeoff," IEEE Transactions on Wireless Communications, vol. 18, no. 1, pp. 241-253, 2018.

[70] R. Correia, N. B. Carvalho, and S. Kawasaki, "Continuously power delivering for passive backscatter wireless sensor networks," IEEE Transactions on Microwave Theory and Techniques, vol. 64, no. 11, pp. 3723-3731, 2016.

[71] D. Belo, R. Correia, P. Pinho, and N. B. Carvalho, "Enabling a constant and efficient flow of wireless energy for iot sensors," in 2017 IEEE MTT-S International Microwave Symposium (IMS). IEEE, 2017, pp. $1342-1344$.

[72] S. D. Assimonis, S.-N. Daskalakis, and A. Bletsas, "Sensitive and efficient rf harvesting supply for batteryless backscatter sensor networks," IEEE Transactions on Microwave Theory and Techniques, vol. 64, no. 4, pp. 1327-1338, 2016.

[73] G. Yang, C. K. Ho, and Y. L. Guan, "Multi-antenna wireless energy transfer for backscatter communication systems," IEEE Journal on Selected Areas in Communications, vol. 33, no. 12, pp. 2974-2987, 2015.

[74] W. Ma, W. Wang, and T. Jiang, "Joint energy harvest and information transfer for energy beamforming in backscatter multiuser networks," IEEE Transactions on Communications, 2020.

[75] X. Tang, G. Xie, and Y. Cui, "Self-sustainable long range backscattering communication using rf energy harvesting," IEEE Internet of Things Journal, 2021.

[76] T.-H. Lin, J. Bito, J. G. Hester, J. Kimionis, R. A. Bahr, and M. M. Tentzeris, "On-body long-range wireless backscattering sensing system using inkjet-/3-d-printed flexible ambient rf energy harvesters capable of simultaneous dc and harmonics generation," IEEE Transactions on Microwave Theory and Techniques, vol. 65, no. 12, pp. 5389-5400, 2017.

[77] I. Sim, Y. M. Hwang, Y. G. Sun, D. I. Kim, Y. Shin, and J. Y. Kim, "Mitigation of phase cancellation for efficient decoding and rf energy harvesting in tag-to-tag communications," IEEE Access, vol. 6, pp. 73 724-73 732, 2018.

[78] Z. Yang, Q. Huang, and Q. Zhang, "Nicscatter: Backscatter as a covert channel in mobile devices," in Proceedings of the 23rd Annual International Conference on Mobile Computing and Networking, 2017, pp. 356-367.

[79] Y. Wang, S. Yan, W. Yang, Y. Huang, and C. Liu, "Energy-efficient covert communications for bistatic backscatter systems," IEEE Transactions on Vehicular Technology, 2021.

[80] Z. Luo, W. Wang, J. Qu, T. Jiang, and Q. Zhang, "Shieldscatter: Improving iot security with backscatter assistance," in Proceedings of the 16th ACM Conference on Embedded Networked Sensor Systems, 2018, pp. 185-198.

[81] J. Y. Han, J. Kim, and S. M. Kim, "Physical layer security improvement using artificial noise-aided tag scheduling in ambient backscatter communication systems," in 2019 Eleventh International Conference on Ubiquitous and Future Networks (ICUFN). IEEE, 2019, pp. 432436.

[82] H. Hassanieh, J. Wang, D. Katabi, and T. Kohno, "Securing rfids by randomizing the modulation and channel," in 12th $\{$ USENIX $\}$ Symposium on Networked Systems Design and Implementation ( $\{$ NSDI $\}$ 15), 2015, pp. 235-249.

[83] Q. Yang, H.-M. Wang, Q. Yin, and A. L. Swindlehurst, "Exploiting randomized continuous wave in secure backscatter communications," IEEE Internet of Things Journal, vol. 7, no. 4, pp. 3389-3403, 2020.
[84] J. Li, C. Wang, A. Li, D. Han, Y. Zhang, J. Zuo, R. Zhang, L. Xie, and Y. Zhang, "Rf-rhythm: Secure and usable two-factor rfid authentication," in IEEE INFOCOM 2020-IEEE Conference on Computer Communications. IEEE, 2020, pp. 2194-2203.

[85] N. Van Huynh, D. N. Nguyen, D. T. Hoang, E. Dutkiewicz, and M. Mueck, "Ambient backscatter: a novel method to defend jamming attacks for wireless networks," IEEE Wireless Communications Letters, vol. 9, no. 2, pp. 175-178, 2019.

[86] D. T. Hoang, D. N. Nguyen, M. A. Alsheikh, S. Gong, E. Dutkiewicz, D. Niyato, and Z. Han, "" borrowing arrows with thatched boats": The art of defeating reactive jammers in iot networks," IEEE Wireless Communications, vol. 27, no. 3, pp. 79-87, 2020.

[87] T. H. Dinh, M. A. Alsheikh, S. Gong, D. Niyato, Z. Han, and Y.C. Liang, "Defend jamming attacks: How to make enemies become friends," in 2019 IEEE Global Communications Conference (GLOBECOM). IEEE, 2019, pp. 1-6.

[88] N. Van Huynh, D. N. Nguyen, D. T. Hoang, and E. Dutkiewicz, ““jjam me if you can:" defeating jammer with deep dueling neural network architecture and ambient backscattering augmented communications," IEEE Journal on Selected Areas in Communications, vol. 37, no. 11, pp. 2603-2620, 2019.

[89] J. Y. Han, M. J. Kim, J. Kim, and S. M. Kim, "Physical layer security in multi-tag ambient backscatter communications-jamming vs. cooperation," in 2020 IEEE Wireless Communications and Networking Conference (WCNC). IEEE, 2020, pp. 1-6.

[90] B. Couraud, T. Deleruyelle, E. Kussener, and R. Vauché, "Real-time impedance characterization method for rfid-type backscatter communication devices," IEEE Transactions on Instrumentation and Measurement, vol. 67, no. 2, pp. 288-295, 2017.

[91] M. A. Varner and G. D. Durgin, "Reflection of modulated radio (remora): Link analysis of ambient scatter radio using perfect pulses," in 2018 IEEE 19th International Workshop on Signal Processing Advances in Wireless Communications (SPAWC). IEEE, 2018, pp. $1-5$.

[92] T. Machii, K. Edamatsu, M. Motoyoshi, S. Kameda, and N. Suematsu, "Link design and received power measurement of $5 \mathrm{ghz}$ band wi-fi backscatter system using a miniaturized antenna module," in 2020 IEEE International Symposium on Radio-Frequency Integration Technology (RFIT). IEEE, 2020, pp. 226-228.

[93] M. Alhassoun and G. D. Durgin, "A theoretical channel model for spatial fading in retrodirective backscatter channels," IEEE Transactions on Wireless Communications, vol. 18, no. 12, pp. 5845-5854, 2019.

[94] A. Cheu, M. M. Morys, C. R. Anderson, and G. D. Durgin, "Rf propagation through vegetation with time-varying moisture," in 2016 IEEE Radio and Wireless Symposium (RWS). IEEE, 2016, pp. 73-75.

[95] D. Darsena, G. Gelli, and F. Verde, "Modeling and performance analysis of wireless networks with ambient backscatter devices," IEEE Transactions on Communications, vol. 65, no. 4, pp. 1797-1814, 2017.

[96] L. Shi, R. Q. Hu, Y. Ye, and H. Zhang, "Modeling and performance analysis for ambient backscattering underlaying cellular networks," IEEE Transactions on Vehicular Technology, vol. 69, no. 6, pp. 65636577, 2020.

[97] X. Gu, L. Grauwin, D. Dousset, S. Hemour, and K. Wu, "Dynamic ambient rf energy density measurements of montreal for battery-free iot sensor network planning," IEEE Internet of Things Journal, 2021.

[98] M. Jin, Y. He, C. Jiang, and Y. Liu, "Parallel backscatter: Channel estimation and beyond," IEEE/ACM Transactions on Networking, 2021.

[99] V. Liu, V. Talla, and S. Gollakota, "Enabling instantaneous feedback with full-duplex backscatter," in Proceedings of the 20th annual international conference on Mobile computing and networking, 2014, pp. $67-78$.

[100] W. Liu, K. Huang, X. Zhou, and S. Durrani, "Full-duplex backscatter interference networks based on time-hopping spread spectrum," IEEE Transactions on Wireless Communications, vol. 16, no. 7, pp. 43614377, 2017.

[101] G. Yang, D. Yuan, Y.-C. Liang, R. Zhang, and V. C. Leung, "Optimal resource allocation in full-duplex ambient backscatter communication networks for wireless-powered iot," IEEE Internet of Things Journal, vol. 6, no. 2, pp. 2612-2625, 2018.

[102] W. Liu, Y.-C. Liang, Y. Li, and B. Vucetic, "On ambient backscatter multiple-access systems," in 2018 IEEE International Conference on Communications (ICC). IEEE, 2018, pp. 1-7.

[103] S. Zeb, Q. Abbas, S. A. Hassan, A. Mahmood, R. Mumtaz, S. H. Zaidi, S. A. R. Zaidi, and M. Gidlund, "Noma enhanced backscatter communication for green iot networks," in 2019 16th International Symposium on Wireless Communication Systems (ISWCS). IEEE, 2019, pp. 640-644. 
[104] J.-H. Kwon, X. Zhang, and E.-J. Kim, "Scalable wi-fi backscatter uplink multiple access for battery-free internet of things," IEEE Access, vol. 9, pp. 30929-30945, 2021.

[105] G. Yang, X. Xu, and Y.-C. Liang, "Resource allocation in nomaenhanced backscatter communication networks for wireless powered iot," IEEE Wireless Communications Letters, vol. 9, no. 1, pp. 117$120,2019$.

[106] Y. Xu, Z. Qin, G. Gui, H. Gacanin, H. Sari, and F. Adachi, "Energy efficiency maximization in noma enabled backscatter communications with qos guarantee," IEEE Wireless Communications Letters, 2020.

[107] A. W. Nazar, S. A. Hassan, H. Jung, A. Mahmood, and M. Gidlund, "Ber analysis of a backscatter communication system with nonorthogonal multiple access," IEEE Transactions on Green Communications and Networking, 2021.

[108] W. U. Khan, X. Li, M. Zeng, and O. A. Dobre, "Backscatter-enabled noma for future $6 \mathrm{~g}$ systems: A new optimization framework under imperfect sic," IEEE Communications Letters, 2021.

[109] M. T. Isik and O. B. Akan, "Padre: modulated backscattering-based passive data retrieval in wireless sensor networks," in 2009 IEEE Wireless Communications and Networking Conference. IEEE, 2009, pp. 1-6.

[110] S. N. Daskalakis, R. Correia, G. Goussetis, M. M. Tentzeris, N. B. Carvalho, and A. Georgiadis, "Four-pam modulation of ambient fm backscattering for spectrally efficient low-power applications," IEEE Transactions on Microwave Theory and Techniques, vol. 66, no. 12 pp. 5909-5921, 2018.

[111] R. Correia and N. B. Carvalho, "Ofdm-like high order backscatter modulation," in 2018 IEEE MTT-S International Microwave Workshop Series on $5 G$ Hardware and System Technologies (IMWS-5G). IEEE, 2018, pp. 1-3.

[112] G. Yang, Y.-C. Liang, R. Zhang, and Y. Pei, "Modulation in the air: Backscatter communication over ambient ofdm carrier," IEEE Transactions on Communications, vol. 66, no. 3, pp. 1219-1233, 2017.

[113] M. A. ElMossallamy, M. Pan, R. Jäntti, K. G. Seddik, G. Y. Li, and Z. Han, "Noncoherent backscatter communications over ambient ofdm signals," IEEE Transactions on Communications, vol. 67, no. 5, pp. 3597-3611, 2019.

[114] J. K. Devineni and H. S. Dhillon, "Manchester encoding for noncoherent detection of ambient backscatter in time-selective fading," IEEE Transactions on Vehicular Technology, 2021.

[115] Y. Zhang, E. Li, Y.-H. Zhu, K. Chi, and X. Tian, "Energy-efficient prefix code based backscatter communication for wirelessly powered networks," IEEE Wireless Communications Letters, vol. 8, no. 2, pp. 348-351, 2018.

[116] S. S. Afzal, R. Ghaffarivardavagh, W. Akbar, O. Rodriguez, and F. Adib, "Enabling higher-order modulation for underwater backscatter communication," in Global Oceans 2020: Singapore-US Gulf Coast. IEEE, 2020, pp. 1-6.

[117] Y. Ma, N. Selby, and F. Adib, "Drone relays for battery-free networks," in Proceedings of the Conference of the ACM Special Interest Group on Data Communication, 2017, pp. 335-347.

[118] S. Yang, Y. Deng, X. Tang, Y. Ding, and J. Zhou, "Energy efficiency optimization for uav-assisted backscatter communications," IEEE Communications Letters, vol. 23, no. 11, pp. 2041-2045, 2019.

[119] G. Yang, R. Dai, and Y.-C. Liang, "Energy-efficient uav backscatter communication with joint trajectory and resource optimization," in ICC 2019-2019 IEEE International Conference on Communications (ICC). IEEE, 2019, pp. 1-6.

[120] Y. Nie, J. Zhao, J. Liu, J. Jiang, and R. Ding, "Energy-efficient uav trajectory design for backscatter communication: A deep reinforcement learning approach," China Communications, vol. 17, no. 10, pp. 129141,2020 .

[121] S. Zhang, W. Wang, N. Zhang, and T. Jiang, "Lora backscatter assisted state estimator for micro aerial vehicles with online initialization," IEEE Transactions on Mobile Computing, 2021.

[122] A. Farajzadeh, O. Ercetin, and H. Yanikomeroglu, "Uav data collection over noma backscatter networks: Uav altitude and trajectory optimization," in ICC 2019-2019 IEEE International Conference on Communications (ICC). IEEE, 2019, pp. 1-7.

[123] Y. Zhang, Z. Mou, F. Gao, L. Xing, J. Jiang, and Z. Han, "Hierarchical deep reinforcement learning for backscattering data collection with multiple uavs," IEEE Internet of Things Journal, 2020.

[124] M. Hua, L. Yang, C. Li, Q. Wu, and A. L. Swindlehurst, "Throughput maximization for uav-aided backscatter communication networks," IEEE Transactions on Communications, vol. 68, no. 2, pp. 1254-1270, 2019.
[125] T. T. Anh, N. C. Luong, and D. Niyato, "A deep reinforcement learning approach for backscatter-assisted relay communications," IEEE Wireless Communications Letters, vol. 10, no. 1, pp. 166-169, 2020.

[126] M. Z. Hassan, M. J. Hossain, J. Cheng, and V. C. Leung, "Statisticalqos guarantee for iot network driven by laser-powered uav relay and rf backscatter communications," IEEE Transactions on Green Communications and Networking, 2020.

[127] X. Jia, J. Zhao, X. Zhou, and D. Niyato, "Intelligent reflecting surfaceaided backscatter communications," in GLOBECOM 2020 - 2020 IEEE Global Communications Conference. IEEE, 2020, pp. 1-6.

[128] J. Choi, "Matched-filter-based backscatter communication for iot devices over ambient ofdm carrier," IEEE Internet of Things Journal, vol. 6, no. 6, pp. 10229-10239, 2019.

[129] Q. Zhang and Y.-C. Liang, "Signal detection for ambient backscatter communications using unsupervised learning," in 2017 IEEE Globecom Workshops (GC Wkshps). IEEE, 2017, pp. 1-6.

[130] Q. Zhang, H. Guo, Y.-C. Liang, and X. Yuan, "Constellation learningbased signal detection for ambient backscatter communication systems," IEEE Journal on Selected Areas in Communications, vol. 37, no. 2 , pp. $452-463,2018$.

[131] A. Rahmati and H. Dai, "Reinforcement learning for interference avoidance game in rf-powered backscatter communications," in ICC 2019-2019 IEEE International Conference on Communications (ICC). IEEE, 2019, pp. 1-6.

[132] W. Zhao, G. Wang, S. Atapattu, T. A. Tsiftsis, and X. Ma, "Performance analysis of large intelligent surface aided backscatter communication systems," IEEE Wireless Communications Letters, vol. 9, no. 7, pp. 962-966, 2020.

[133] G. Yang, Q. Zhang, and Y.-C. Liang, "Cooperative ambient backscatter communications for green internet-of-things," IEEE Internet of Things Journal, vol. 5, no. 2, pp. 1116-1130, 2018.

[134] T. Liu, X. Qu, W. Tan, and Y. Cheng, "An energy efficient cooperative communication scheme in ambient rf powered sensor networks," IEEE Access, vol. 7, pp. 86545-86554, 2019.

[135] W. Chen, H. Ding, S. Wang, D. B. da Costa, F. Gong, and P. H. J. Nardelli, "Backscatter cooperation in noma communications systems," IEEE Transactions on Wireless Communications, 2021.

[136] L. X. Nguyen, C. T. Nguyen, V. X. Phan, and A. T. Pham, "A novel user pairing scheme for non-orthogonal multiple access backscatter communication," in 2020 IEEE Eighth International Conference on Communications and Electronics (ICCE). IEEE, 2021, pp. 509-514.

[137] J. Essel, D. Brenk, J. Heidrich, and R. Weigel, "A highly efficient uhf rfid frontend approach," in 2009 IEEE MTT-S International Microwave Workshop on Wireless Sensing, Local Positioning, and RFID. IEEE, 2009, pp. 1-4.

[138] S. Rana, T. Prabhakar, and K. Vinoy, "An efficient architecture for battery-less terminals for internet of things," in 2015 IEEE Applied Electromagnetics Conference (AEMC). IEEE, 2015, pp. 1-2.

[139] P. Portos, P. Ratajczak, and F. Ferrero, "Design of a miniature and reconfigurable antenna system for spatial modulation and ambient backscattering," in 2020 International Workshop on Antenna Technology $(i W A T)$. IEEE, 2020, pp. 1-3.

[140] D. Matos, R. Correia, and N. Carvalho, "mm-wave backscatter frontend for 5g-iot/wpt applications," in 2020 IEEE International Electron Devices Meeting (IEDM). IEEE, 2020, pp. 37-7.

[141] D. Liu, X. Sun, J. Wang, and F. Kang, "Implementation of an energyefficient digital baseband controller compatible with epc class-1 gen2 standard," in 2017 IEEE 12th International Conference on ASIC (ASICON). IEEE, 2017, pp. 387-390.

[142] J. D. Rosenthal and M. S. Reynolds, "Hardware-efficient all-digital architectures for ofdm backscatter modulators," IEEE Transactions on Microwave Theory and Techniques, 2020.

[143] P. Zhang, P. Hu, V. Pasikanti, and D. Ganesan, "Ekhonet: High speed ultra low-power backscatter for next generation sensors," in Proceedings of the 20th annual international conference on Mobile computing and networking, 2014, pp. 557-568.

[144] Y. Tao, B. Li, C. Zhao, and Y.-C. Liang, "Hardware-efficient signal detection for ambient backscattering communications," IEEE Соттиnications Letters, vol. 23, no. 12, pp. 2196-2199, 2019.

[145] P. Zhang and D. Ganesan, "Enabling bit-by-bit backscatter communication in severe energy harvesting environments," in 11th \{USENIX\} Symposium on Networked Systems Design and Implementation ( $\{$ NSDI\} 14), 2014, pp. 345-357.

[146] X. Li, Y. Zheng, W. U. Khan, M. Zeng, D. Li, L. Li et al., "Physical layer security of cognitive ambient backscatter communications for green internet-of-things," IEEE Transactions on Green Communications and Networking, 2021. 
[147] M. Rostami, K. Sundaresan, E. Chai, S. Rangarajan, and D. Ganesan, "Redefining passive in backscattering with commodity devices," in Proceedings of the 26th Annual International Conference on Mobile Computing and Networking, 2020, pp. 1-13.

[148] Y. Ding, G. Goussetis, R. Correia, R. Lihakanga, N. B. Carvalho, and $\mathrm{P}$. Petridis, "Enabling multicarrier backscattering communications," in 2020 IEEE MTT-S International Wireless Symposium (IWS). IEEE, 2020, pp. 1-3.

[149] Q. Tao, Y. Li, C. Zhong, S. Shao, and Z. Zhang, "A novel interference cancellation scheme for bistatic backscatter communication systems," IEEE Communications Letters, 2021.

[150] X. Wen, S. Bi, X. Lin, L. Yuan, and J. Wang, "Throughput maximization for ambient backscatter communication: A reinforcement learning approach," in 2019 IEEE 3rd Information Technology, Networking, Electronic and Automation Control Conference (ITNEC). IEEE, 2019, pp. 997-1003.

[151] Q. Huang, Y. Mei, W. Wang, and Q. Zhang, "Battery-free sensing platform for wearable devices: The synergy between two feet," in IEEE INFOCOM 2016-The 35th Annual IEEE International Conference on Computer Communications. IEEE, 2016, pp. 1-9.

[152] A. Varshney, A. Soleiman, and T. Voigt, "Tunnelscatter: Low power communication for sensor tags using tunnel diodes," in The 25th Annual International Conference on Mobile Computing and Networking, 2019, pp. 1-17.

[153] A. Varshney and L. Corneo, "Tunnel emitter: tunnel diode based low-power carrier emitters for backscatter tags," in Proceedings of the 26th Annual International Conference on Mobile Computing and Networking, 2020, pp. 1-14.

[154] R. Duan, X. Wang, H. Yigitler, M. U. Sheikh, R. Jantti, and Z. Han, "Ambient backscatter communications for future ultra-low-power machine type communications: Challenges, solutions, opportunities, and future research trends," IEEE Communications Magazine, vol. 58, no. 2, pp. 42-47, 2020.

[155] S. J. Nawaz, S. K. Sharma, B. Mansoor, M. N. Patwary, and N. M Khan, "Non-coherent and backscatter communications: Enabling ultramassive connectivity in $6 \mathrm{~g}$ wireless networks," IEEE Access, 2021.

[156] T. O. Timoudas, R. Du, and C. Fischione, "Enabling massive iot in ambient backscatter communication systems," in ICC 2020-2020 IEEE International Conference on Communications (ICC). IEEE, 2020, pp. $1-6$. 